\title{
The Effect of the Madden-Julian Oscillation on the North Atlantic Oscillation Using Idealized Numerical Experiments
}

\author{
SEBASTIEN FROMANG ${ }^{\mathrm{a}}$ \\ Laboratoire AIM, CEA/DSM-CNRS-Université Paris 7, Irfu/Departement d'Astrophysique, Gif-sur-Yvette, France \\ GWENDAL RIVIÈRE \\ LMD/IPSL, École Normale Supérieure, PSL Université, École Polytechnique, Institut Polytechnique de Paris, \\ Sorbonne Université, CNRS, Paris, France
}

(Manuscript received 4 July 2019, in final form 9 December 2019)

\begin{abstract}
The aim of the paper is to investigate the influence of the Madden-Julian oscillation (MJO) on the North Atlantic Oscillation (NAO) using a quasigeostrophic model on the sphere. A simplified forcing based on potential vorticity anomalies in the tropics is used to mimic the MJO. The idealized nature of our setup allows us to determine the distinct roles played by stationary and synoptic waves. This is done by means of several series of almost 10000 short runs of 30 days. Ensemble averages and a streamfunction budget analysis are used to study the modifications of the flow induced by the MJO. We find that a stationary Rossby wave is excited in the tropics during MJO phase 3. The western part of the Pacific jet is displaced poleward, which modifies the transient eddy activity in that basin. These changes create a ridge south of Alaska, which favors equatorward propagation of synoptic waves and larger poleward eddy momentum fluxes from the eastern Pacific toward the Atlantic, increasing the frequency of occurrence of the positive NAO events. The situation is essentially reversed following phase 6 of the MJO and conducive to the negative phase of the NAO. For a realistic MJO forcing amplitude, we find increases in both NAO phases to be around $30 \%$, in reasonable agreement with the observations given the model simplicity. Finally, we present a series of experiments to assess the relative importance of linear versus nonlinear effects.
\end{abstract}

\section{Introduction}

The extratropical atmospheric circulation is significantly influenced by the tropical modes of variability at various time scales. For example, on seasonal, interannual, and even longer time scales, the influence of El Niño-Southern Oscillation (ENSO) on the midlatitudes has been widely studied (Bjerknes 1966; Hoerling and Kumar 2002): ENSO is known to affect various aspects of the extratropical climate, such as extreme precipitation and temperature anomalies over the United States (Gershunov and Barnett 1998; Cayan et al. 1999), midlatitude cyclogenesis (Schemm et al. 2018), midlatitude

\footnotetext{
${ }^{\text {a }}$ Current affiliation: Laboratoire des Sciences du Climat et de l'Environnement, LSCE/IPSL, CEA-CNRS-UVSQ, Université Paris-Saclay, F-91191, Gif-sur-Yvette, France.
}

Corresponding author: Sebastien Fromang, sebastien.fromang@ cea.fr atmospheric low-frequency variability (L'Heureux and Thompson 2006). Its influence also extends to polar regions (Yuan et al. 2018) as well as to the stratosphere (Domeisen et al. 2019). On intraseasonal time scales, there has recently been a growing interest on the influence of the Madden-Julian oscillation (MJO; Madden and Julian 1971) on various aspects of the midlatitude climate (Stan et al. 2017). This is partly motivated by the possibility of improving the medium-range time-scale predictability at extratropical latitudes with lead times of up to 2 weeks. The MJO is indeed the dominant source of intraseasonal variability in the tropics and, as such, might be an important driver of the midlatitude circulation. Although a detailed understanding of its dynamical origin is still lacking and is a subject of active research, its properties are well documented [see Zhang (2005) for a review]. It consists in a dipole of enhanced/suppressed convection with a typical zonal scale of $12000-20000 \mathrm{~km}$ (corresponding to $100^{\circ}-180^{\circ}$ in longitudinal extent) that moves eastward with a typical velocity of order $5 \mathrm{~m} \mathrm{~s}^{-1}$. 
It first appears in the Indian Ocean and moves across the Maritime Continent toward the Pacific Ocean where it gradually weakens over a time scale of 40-50 days.

The MJO convective activity creates circulation anomalies in the tropics within a few days that extends to the extratropics in a couple of weeks (Matthews et al. 2004), affecting the climate at remote locations, not only in the tropics, but also at extratropical and polar latitudes. In the tropics, seasonal modulations of the precipitation in Mexico (Perdigón-Morales et al. 2019) and in the greater Caribbean (Curtis and Gamble 2016) have been reported in relation to the MJO. Over the polar regions, it has been found to alter the frequency of occurrence of stratospheric sudden warming (Garfinkel et al. 2012), an effect that might well amplify in the future (Kang and Tziperman 2018). The MJO influence in the Arctic also has a significant impact in the troposphere: it was indeed found to affect polar surface temperature variability (Lee et al. 2011) and sea ice extent (Henderson et al. 2014). But perhaps the most widely studied of these relationships concern the consequences of the MJO for the midlatitude tropospheric circulation. Several observational studies have shown that it affects the Pacific jet and its associated storm tracks (Matthews and Kiladis 1999; Moore et al. 2010; Sakaeda and Roundy 2014; Guo et al. 2017). In general, the nature of these modifications vary during the MJO life cycle. This life cycle is usually divided into eight phases that correspond to different locations of the anomalies (Wheeler and Hendon 2004): there is enhanced/reduced convection over the Indian Ocean/western Pacific Ocean during phases 1-3 of the MJO, with the opposite situation occurring during MJO phases 6-7. Phase 4 and 5 correspond to intermediate situations for which the site of enhanced convection is found over the Maritime Continent. Typically, the 200-mb Pacific midlatitude jet $(1 \mathrm{mb}=1 \mathrm{hPa})$ is found to shift poleward (equatorward) during phases 1-3 (phases 6-8) of the MJO (Moore et al. 2010), with similar shift observed for the associated storm tracks (Zheng et al. 2018). The influence of the MJO extends to hemispheric scales and is known to alter the Artic Oscillation (L'Heureux and Higgins 2008). Maybe what is surprising is the fact that it also affects the Euro-Atlantic sector midlatitude circulation far away from its source region. Cassou (2008) and Lin et al. (2009) indeed reported a lagged correlation between the North Atlantic Oscillation (NAO) and specific phases of the MJO: the positive (negative) phase of the NAO, characterized by a poleward (equatorward) shift of the North Atlantic jet, is found to occur with an excess frequency of up to 50\% 10-15 days after phase 3 (phase 6) of the MJO. This result has since been confirmed by different teams (Frederiksen and Lin 2013; Henderson et al. 2016; Jiang et al. 2017) and its dynamical understanding constitutes the focus of the present paper.

In general, the MJO-extratropical relationships described above are believed to be mediated by the excitation of stationary Rossby waves triggered by the convective upper-tropospheric divergent flow (Sardeshmukh and Hoskins 1988). These waves propagate to the midlatitudes along great circles (Hoskins and Karoly 1981; Hoskins and Ambrizzi 1993) over a typical time scale of 2 weeks (Jin and Hoskins 1995). Several theoretical studies based on general circulation models (GCMs) indeed demonstrated that the MJO excites such a wave train (Matthews et al. 2004; Lin et al. 2010; Seo and Son 2012; Goss and Feldstein 2015; Seo et al. 2016; Goss and Feldstein 2017, 2018; Lukens et al. 2017). However, these studies, which did not directly address the MJO-NAO relationship, generally analyzed the effect of the MJO on a stationary climatological flow, thereby ignoring the modulating role of transients, despite their known important role in determining the flow extratropical response (Held et al. 1989). They also considered fixed heating forcing as a proxy to model the MJO. Such limitations have been relaxed in recent years. For example, using a fully coupled ocean-atmosphere model and adding a composite eastward-moving MJO heating, Straus et al. (2015) recovered an excess occurrence of $\mathrm{NAO}+$ events lagging $\mathrm{MJO}$ phase 3 , and pointed to the synchronous role of the stationary Rossby wave and synoptic eddy fluctuations in shaping the atmospheric response over the Atlantic. In agreement with the early suggestion of Cassou (2008), the picture that thus emerges from that work is that of a complicated interplay between the MJO excited stationary Rossby wave and the nonlinear synoptic transient eddy activity in the atmosphere. More recently, these complex model calculations have been complemented by more idealized dry GCM calculations that confirm the importance of transient eddies but give a conflicting interpretation regarding the relationship between the MJO and the NAO. Shao et al. (2019) used a series of short GCM calculations forced with a fixed heating as a model of MJO phase 3 and showed that they also recovered the lagged correlation with the positive phase of the NAO in this simpler framework. The excess occurrence of the $\mathrm{NAO}+$ is attributed to an anomalous synoptic activity over the Atlantic. However, the precise origin of these anomalies and their relationship with the MJO excited stationary Rossby wave over the Pacific remains unclear. By contrast, and while using a similar method, Lin and Brunet (2018) favored a different interpretation to explain the occurrence of the NAO+ that follows phase 3 of the MJO: instead of relying on a modified synoptic activity in the Atlantic region, they 
instead demonstrate an early influence of a westwardpropagating Rossby wave excited by the MJO that projects positively on the NAO + . This discussion shows that the respective role of the stationary and transient eddies remains to be clarified. This is the main goal of this paper. To do so, we adopt an even simpler approach by using a highly idealized, low-resolution, quasigeostrophic (QG) model. Thanks to its low computational cost, this allows us to explore the sensitivity of the atmospheric response to the MJO more systematically and with a higher significance compared to these earlier approaches.

The plan of the paper is as follows. In section 2, we describe the numerical method we used and the different experiments we performed. The main results are presented in section 3 and the physical mechanisms are elucidated in section 4 . Sensitivity experiments are then detailed in section 5. We finally summarize our results and discuss their limitations in section 6 .

\section{Method}

\section{a. Quasigeostrophic code}

We use the QG spectral model on the sphere introduced by Marshall and Molteni (1993). It is based on the potential vorticity (PV) evolution at three pressure levels, namely, 200, 500, and $800 \mathrm{mb}$, according to the equation

$$
\frac{\partial q_{i}}{\partial t}=-J\left(\psi_{i}, q_{i}\right)-D_{i}\left(\psi_{1}, \psi_{2}, \psi_{3}\right)+S_{i}
$$

where $J$ is the Jacobian operator and $S_{i}$ is a spatially varying but time-independent forcing term that is discussed in more details in the following sections. The variables $q_{i}$ and $\psi_{i}$ are the PV and streamfunction at each level $i$, respectively. They are related through

$$
\begin{aligned}
& q_{1}=f+\nabla^{2} \psi_{1}-R_{01}^{2}\left(\psi_{1}-\psi_{2}\right), \\
& q_{2}=f+\nabla^{2} \psi_{2}+R_{01}^{2}\left(\psi_{1}-\psi_{2}\right)-R_{02}^{2}\left(\psi_{2}-\psi_{3}\right), \\
& q_{3}=f\left(1+\frac{h}{H_{0}}\right)+\nabla^{2} \psi_{3}+R_{02}^{2}\left(\psi_{2}-\psi_{3}\right) .
\end{aligned}
$$

In these relations, $f=2 \Omega \sin \phi$ is the Coriolis parameter, in which $\Omega$ is Earth's rotation rate. $R_{01}=700 \mathrm{~km}$ and $R_{02}=450 \mathrm{~km}$ are respectively the deformation radii of the 200-500- and 500-800-mb layers, $h$ is a realistic topographic altitude, and $H_{0}=9 \mathrm{~km}$ is a scaling parameter. Defining $q_{i}^{\prime}=q_{i}-f$, the dissipation term $D_{i}$ is written according to

$$
\begin{aligned}
& D_{1}=c_{H} \nabla^{8} q_{1}^{\prime}-\tau_{R}^{-1} R_{01}^{2}\left(\psi_{1}-\psi_{2}\right) \\
& D_{2}=c_{H} \nabla^{8} q_{2}^{\prime}+\tau_{R}^{-1} R_{01}^{2}\left(\psi_{1}-\psi_{2}\right)-\tau_{R}^{-1} R_{02}^{2}\left(\psi_{2}-\psi_{3}\right) \\
& D_{3}=c_{H} \nabla^{8} q_{3}^{\prime}+\tau_{R}^{-1} R_{02}^{2}\left(\psi_{2}-\psi_{3}\right)-\tau_{E}^{-1} \nabla^{2} \psi_{3}
\end{aligned}
$$

At each level, the different terms respectively correspond to a small-scale hyperdiffusion, a temperature relaxation with time scale $\tau_{R}=25$ days and finally a linear drag with time scale $\tau_{E}$ at $800 \mathrm{mb}$.

All the parameters we used in this paper have the same numerical values as in Drouard et al. (2013). The only exception is $\tau_{E}$, which we set to 2 days because of the better agreement we obtained with the observed climatology when using that value. A T42 truncation is used throughout the paper.

\section{b. Forcing}

We used two different forcing $S_{i}$. The first is designed so that the flow climatology is close to the observations and will be denoted $S_{i}^{\mathrm{clim}}$. Marshall and Molteni (1993) suggested a method to calculate $S_{i}^{\text {clim }}$ based on the combination of the observed daily PV and streamfunction, an idea that was later used for the case of dry GCM applications (Hall 2000). The obtained climatology is in qualitative agreement with the observations but displays significant quantitative differences (Drouard et al. 2013). This is unfortunate for the purpose of this paper because the background flow strongly influences the propagation of stationary Rossby waves (Hoskins and Karoly 1981), which, as discussed in the introduction, are believed to be an important piece of the interaction between the MJO and the midlatitudes. An alternative strategy is to use an iterative method to evaluate an appropriate forcing. This has been used in the context of dry GCM models by several authors: assuming that the forcing function takes the form of a thermal relaxation, an algorithm iteratively calculates the equilibrium temperature such that the model climatology approaches a given target (Lunkeit et al. 1998; Chang 2006; Yuval and Kaspi 2016). ${ }^{1}$ The method we developed builds on these ideas but is slightly different. No assumption is made on the explicit form of $S_{i}^{\text {clim }}$. A Newton-Krylov iterative method is used to evaluate the most appropriate forcing function: starting from an initial guess, the algorithm gradually modifies $S_{i}^{\text {clim }}$ so that the model climatology matches an observed climatology, which is here the ERA-Interim wintertime (December-February) climatological mean over the period 1979-2016. The algorithm is briefly

\footnotetext{
${ }^{1}$ Note that the forcing function is time dependent in that case.
} 

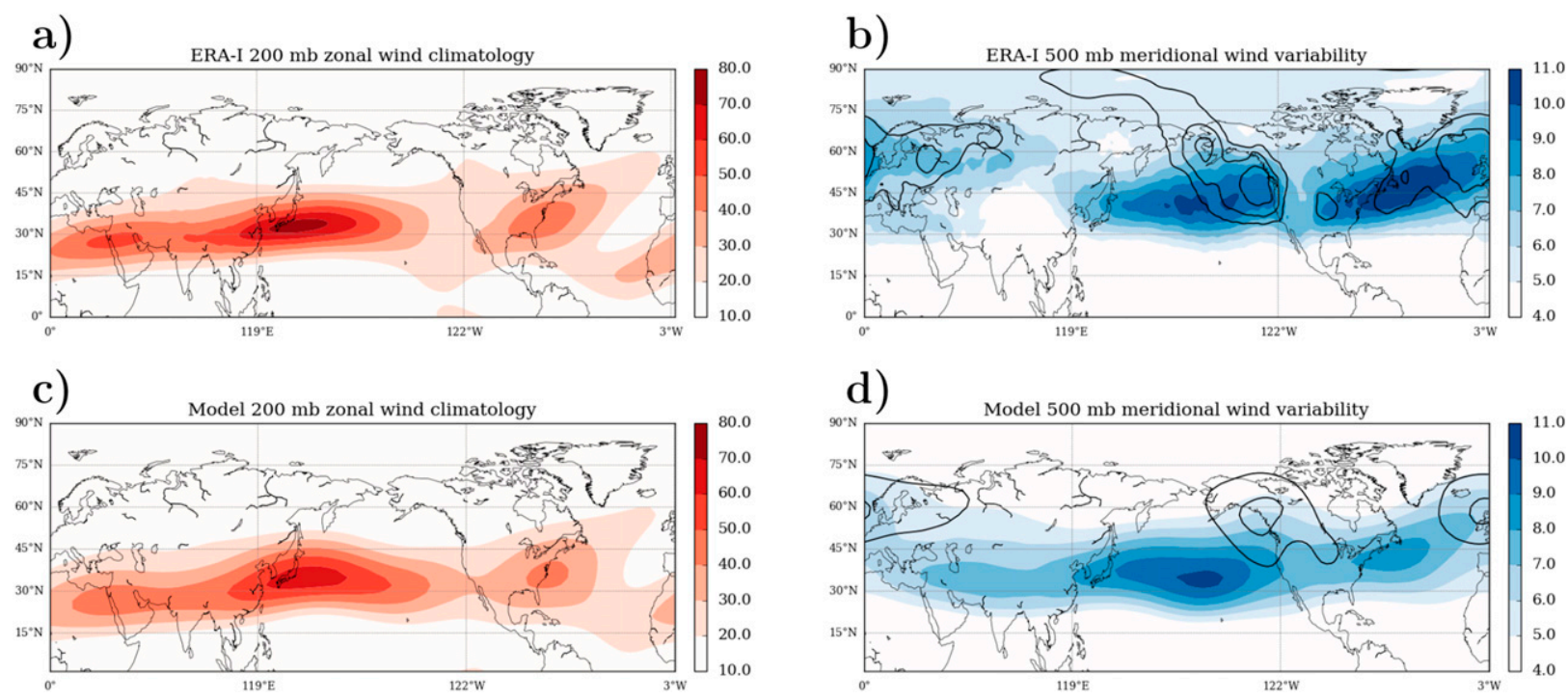

FIG. 1. (left) The 200-mb zonal wind climatology from (a) ERA-Interim and (c) the 300000 -day-long simulation and (right) the 500-mb meridional wind variability from (b) ERA-Interim and (d) the 300000-day-long simulation. Plotted in (b) and (d) are the standard deviations of the high-frequency (shading) and low-frequency (contours) components. Contours are plotted at $7.5,8.5$, and $9.5 \mathrm{~m} \mathrm{~s}^{-1}$. Shading units are $\mathrm{m} \mathrm{s}^{-1}$ in all panels.

described and quantitatively validated in the appendix. Using the obtained forcing function, we next performed a perpetual-winter 300000 -day-long run (i.e., roughly 800 years), which will constitute our control simulation in the following. Excluding the first 1000 days of that run, we used daily data to compare the simulated 200-mb zonal wind climatology and the 500-mb meridional wind variability with ERA-Interim data. The atmosphere variability is evaluated by calculating the convolution product of the meridional wind $v$ with a Lanczos filter (Lanczos 1988) with a cutoff period of 8 days so as to write it as the sum of its time-averaged, low- and high-frequency components

$$
v(t)=\bar{v}^{\mathrm{REF}}+v^{L}(t)+v^{H}(t) .
$$

The climatology of the 200-mb zonal wind (labeled $\bar{u}^{\mathrm{REF}}$ ) and the standard deviation of the low- and highfrequency components of the 500-mb meridional wind are compared with the observed fields in Fig. 1. The main features of the Northern Hemisphere atmospheric circulation are recovered. Of particular interest is that the North Pacific and North Atlantic 200-mb jets start to be separated in the model, a property that is poorly captured when using the method of Marshall and Molteni (1993) to estimate the forcing (Drouard et al. 2013). The zonal wind climatology at 500 and $800 \mathrm{mb}$ (not shown) are also in broad agreement with the observations, although we found that the zonal wind at the lowest level always tends to be overestimated in the model. As a result, the baroclinicity of the midlatitude jets is reduced, which probably explains why we find reduced standard deviations of the meridional wind at $500 \mathrm{mb}$ in the model compared to ERA-Interim (cf. Figs. 1b and 1d). Nevertheless, we also find a clear separation between the Pacific and Atlantic storm tracks (as seen from the high-frequency component of the meridional wind), as well as two well-defined maxima of the low-frequency activity located south of Alaska and over northern Europe. Both features nicely mirror the same aspects seen in the observed fields, although the simulated low-frequency activity in the eastern Pacific is slightly shifted northeastward compared to the observed variability. The model results also show significantly more variability over the Himalayas compared to ERA-Interim data. Corti et al. (1997) and Drouard et al. (2013) showed that the NAO was realistically simulated by the model using Marshall and Molteni's (1993) forcing (not shown). As will become clear in section 3, this is also the case when using this new forcing.

Throughout this paper, we will also use the forcing $S_{i}^{\text {stat }}$ that is defined according to the following relation:

$$
S_{i}^{\mathrm{stat}}=J\left(\psi_{i}^{\mathrm{REF}}, q_{i}^{\mathrm{REF}}\right)+D_{i}\left(\psi_{1}^{\mathrm{REF}}, \psi_{2}^{\mathrm{REF}}, \psi_{3}^{\mathrm{REF}}\right) .
$$

The control experiment flow climatology is an exact solution of Eq. (1) when $S_{i}=S_{i}^{\text {stat }}$, which means that only the perturbations with respect to $q_{i}^{\mathrm{REF}}$ and $\psi_{i}^{\mathrm{REF}}$ evolve with time in that case. In the following, we will contrast the results obtained using $S_{i}^{\text {clim }}$ and $S_{i}^{\text {stat }}$ to understand the role of the background flow variability in the lagged relationship between the MJO and the NAO. 


\section{c. Modeling the $\mathrm{MJO}$}

The atmosphere equatorial dynamics is not well captured in the QG approximation (Mak 1991) and the MJO does not naturally develop in our model. For these reasons, we designed a procedure that models its effect on the PV at the vicinity of the equator by means of an additional forcing. Its form was motivated by the properties of the MJO (vorticity perturbations close to the convective center, zonal propagation velocity) and its effect on the midlatitudes. Our main focus was to reproduce qualitatively the phase and wavelength of the eastward-propagating Rossby wave in the midlatitude Pacific area and its associated archlike pattern. We also tuned the forcing amplitude so that the perturbations it creates over that region falls within the range of the observed anomalies. However, the artificial nature of our approach refrained us from making a quantitative and detailed comparison with the observations. This should be the focus of future work, perhaps using a GCM that would be better suited at capturing the equatorial dynamics involved. Below, we detail the line of reasoning we followed to model the MJO within the framework of our QG code.

The MJO is known to be associated with very typical structures (Zhang 2005; Kiladis et al. 2005): in the upper troposphere, two cyclones (anticyclones) are found straddling the equator west (east) of the enhanced convection center. The situation is opposite in the lower troposphere, that is, cyclones to the east of the convection and anticyclones to the west. We can model these features by adding a forcing $S_{i}^{\mathrm{MJO}}$ to $S_{i}$ that takes nonvanishing values only over a region of finite meridional and zonal extent. It is defined according to

$$
S_{i}^{\mathrm{MJO}}=\varepsilon_{i} S_{0} f(\lambda) g(\phi),
$$

where $f(\lambda)$ and $g(\phi)$ are two dimensionless functions of longitude $\lambda$ and latitude $\phi$, respectively:

$$
f(\lambda)=\left\{\begin{array}{cl}
\sin \left[\pi \frac{\lambda-\lambda_{0}(t)}{\Delta \lambda_{0}}\right] & \text { if }\left|\lambda-\lambda_{0}(t)\right|<\Delta \lambda_{0}, \\
0 & \text { otherwise }
\end{array}\right.
$$

and

$$
g(\phi)=\left\{\begin{array}{cl}
\left(\cos \left[\frac{\pi}{2}\left(\frac{\phi}{\Delta \phi_{0}}-1\right)\right]\right)^{2} & \text { if }|\phi|<2 \Delta \phi_{0} \\
0 & \text { otherwise. }
\end{array}\right.
$$

In the above equations $\Delta \phi_{0}$ and $\Delta \lambda_{0}$ represent the typical size of the region where the PV is directly influenced by the MJO. They also affect the longitudinal and meridional extents of the midlatitude response to the forcing. Empirically, we found that using $\Delta \phi_{0}=17.5^{\circ}$ and $\Delta \lambda_{0}=$ $80^{\circ}$ results in an eastward-propagating Rossby wave train that qualitatively resembles that seen in the observations in the Pacific region as seen for example in Fig. 3 of Henderson et al. (2016) or in Fig. 4 of Seo and Son (2012). In Eq. (10), $\varepsilon_{i}$ is a dimensionless parameter that characterizes the vertical profile of the forcing and is such that

$$
\varepsilon_{1}=1, \quad \varepsilon_{2}=0, \quad \text { and } \quad \varepsilon_{3}=-1 / 10 .
$$

These values are motivated by the fact that the MJO heating of the atmosphere is largest in the midtroposphere (Matthews et al. 2004) and thus induces vorticity anomalies of opposite sign in the upper and lower troposphere (Gill 1980; Zhang 2005; Seo and Son 2012). We used a smaller absolute value for $\varepsilon_{3}$ compared to $\varepsilon_{1}$ to reflect the baroclinic nature of the MJO heating, which tends to induce weaker circulation anomalies in the lower troposphere than in the upper troposphere (Seo and Son 2012). The precise value of their ratio was determined by trial and error but is essentially ad hoc.

In Eq. (10) $S_{0}$ is the only dimensional parameter and corresponds to the MJO forcing amplitude. As explained above, we chose its value so that the midlatitude perturbations in the Pacific area have a reasonable amplitude compared to the observations. We will also investigate the sensitivity of our results to varying $S_{0}$ in section $2 \mathrm{~b}$, where we consider in particular the case of very small amplitudes. When $S_{0}$ is positive (negative), $\lambda_{0}(t)$ corresponds to the longitude of enhanced (reduced) convection. It is defined according to

$$
\lambda_{0}(t)=\lambda_{\mathrm{MJO}}^{0}+c_{\mathrm{MJO}} t,
$$

where $c_{\mathrm{MJO}}$ corresponds to the eastward velocity of the MJO and $\lambda_{\text {MJO }}^{0}$ denotes the location of the MJO convective center at $t=0$. In this work, we used $\left(\lambda_{\mathrm{MJO}}^{0}, c_{\mathrm{MJO}}\right)=\left(60^{\circ}, 4.2^{\circ} \mathrm{day}^{-1}\right)$. With these parameters, the MJO is initially located over the Indian Ocean and moves eastward by $100^{\circ}$ in 24 days with a velocity roughly in agreement with the observed value of $5 \mathrm{~m} \mathrm{~s}^{-1}$ (Zhang 2005). After about 15 days, cases with positive $S_{0}$ values resemble the situation that occurs during phase 3 of the MJO (with earlier times corresponding to phases 1 and 2 ), while negative $S_{0}$ values resemble phase 6 of the MJO. In the remainder of this paper, we will therefore refer to the former as "phase $3 \mathrm{MJO}$ forcing" and to the latter as "phase $6 \mathrm{MJO}$ forcing." Finally, the results we obtained are contrasted with experiments with a fixed MJO forcing $\left(c_{\mathrm{MJO}}=0 \mathrm{~m} \mathrm{~s}^{-1}\right)$ located at an intermediate position between its most eastern and western positions in the eastward-moving set of experiments. We thus used 
$\lambda_{\text {MJO }}^{0}=110^{\circ}$ in that case, which roughly corresponds to the location of the MJO forcing at $t=15$ days in the eastward-moving $\mathrm{MJO}$ forcing cases.

\section{d. Numerical experiments}

In this paper, we present the results of two types of numerical experiments. First, we used $S_{i}=S_{i}^{\mathrm{clim}}+S_{i}^{\mathrm{MJO}}$ and refer below to these runs as "time-varying reference flows" experiments. When $S_{0}>0$, the anomalies that emerge combine the direct response of the flow to the MJO forcing but also the modifications to the reference flow that are induced by this direct response. The difficulty is that the anomalies associated with the direct response to the MJO are smaller in amplitude than the internal fluctuations of the atmosphere. To overcome that problem, we performed an ensemble of short-term simulations starting from different initial conditions and averaged the results to extract the composite response of the atmosphere: for that purpose, we restarted our control simulation every 30 days from $t=1000$ days onward with a nonvanishing MJO forcing amplitude to produce an ensemble of 9967 independent shorts runs. Each short run has a duration of 34 days, the last 4 days of which are only used for filtering purposes. Because of the large size of this ensemble, the climatology of the anomalies we obtain is robust. In fact, the lowest contours of all the streamfunction anomalies we show in the remainder of this paper are all significant at the level of at least $99.9 \%$ according to a $t$ test. Second, we used $S_{i}=S_{i}^{\mathrm{stat}}+S_{i}^{\mathrm{MJO}}$ and initialized the flow with the climatology of the control simulation so that it remains stationary for vanishing MJO forcing amplitudes. When $S_{0}>0$, the perturbations that emerge are solely a result of the MJO forcing. We will refer to these runs as "stationary reference flow" experiments.

\section{Results}

\section{a. Eastward-moving phase 3 MJO forcing}

\section{1) Streamfunction ANOMALies}

We first consider the case of phase $3 \mathrm{MJO}$ forcing for which $S_{0}=4 \times 10^{-11} \mathrm{~s}^{-2}$ and compare the time evolution of the 200-mb streamfunction anomalies for both the fixed and time-varying reference flow cases in Fig. 2 (left and right columns, respectively). As explained above, the latter case is obtained after an ensemble average over the 9967 members of the series.

Five days after the MJO forcing is turned on, a dipolar anomaly is observed in the western Pacific ocean (Figs. 2a,b). It consists in a trough and a ridge located at a latitude of $+15^{\circ}$ and $+40^{\circ}$, respectively. The trough is seen to be in phase with the MJO forcing (shown with red contours). In agreement with previously published results (Hoskins and Ambrizzi 1993; Seo and Son 2012), the flow response to the MJO perturbation is largely barotropic in nature in the extratropics (not shown) and is almost identical in the two cases (cf. Figs. 2a and 2b).

After 15 days (Figs. 2c,d), streamfunction anomalies are found as far as the northern Atlantic basin and take the form of a large-scale quasi-stationary Rossby wave. In agreement with previous results (Hoskins and Jin 1991; Ambrizzi and Hoskins 1997), it has an equivalent barotropic structure (not shown) with no indication of baroclinic modes growing in the midlatitude regions in the stationary reference flow experiment. In general, for a given MJO forcing amplitude, we have found that its amplitude is smaller in the time-varying reference flow case than in the stationary reference flow case (cf. left and right columns). In section 4, we will show that this can be understood as arising from increased nonlinear interactions induced by the low-frequency variability of the reference flow. Maybe more interestingly, at $t=15$ days, the streamfunction anomaly spatial structure depends on the nature of the reference flow: while it displays an archlike pattern for the stationary reference flow case, as reported in various papers using a similar setup (Matthews et al. 2004; Seo and Son 2012; Seo et al. 2016), it is more zonally elongated when the reference flow is time varying. For example, the midlatitude anticyclonic anomaly (located between $30^{\circ}$ and $45^{\circ} \mathrm{N}$ ) extends only to about $180^{\circ}$ in longitude for the stationary reference flow case but all the way to the western coast of North America $\left(\lambda \sim 110^{\circ} \mathrm{W}\right)$ for the time-varying reference flow case. In addition, the trough located above Alaska has weakened and its southeastward extension along the western coast of North America has disappeared. Similarly, the ridge located over Canada has significantly weakened for the time-varying flow case and is barely visible with the contour levels used in Fig. 2. The zonal wind anomalies are on the order of a few meters per second (black contours) and correspond in both cases to a poleward displacement of the midlatitude Pacific jet. In agreement with the streamfunction anomalies, it is confined to the western Pacific for the case of the stationary reference flow, while it extends all the way to the eastern Pacific basin in the time-varying reference flow case.

After 25 days (Figs. 2e,f), the differences between the two types of experiments have increased: when the reference flow is time varying, a strong ridge has emerged south of Alaska, while this feature is completely absent from the stationary reference flow experiments. The appearance of such a ridge in the eastern Pacific is qualitatively similar to that seen in reanalysis data [see, e.g., pentads 2-3 in Fig. 4 of Henderson et al. (2016)]. By contrast, at earlier time, the same reanalysis data 
a)

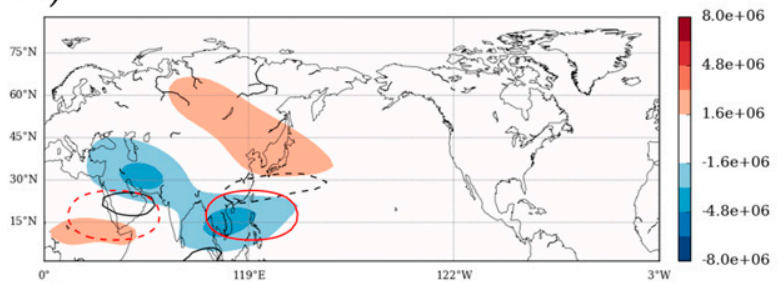

c)

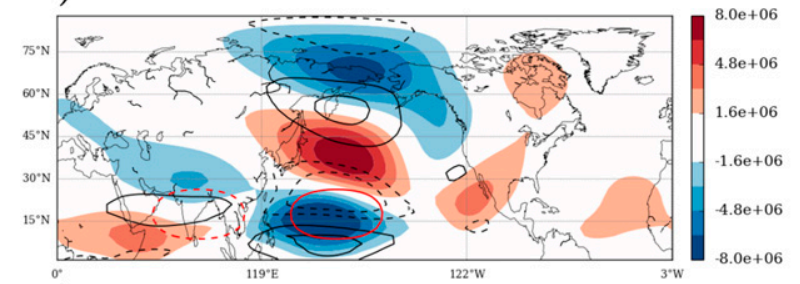

e)

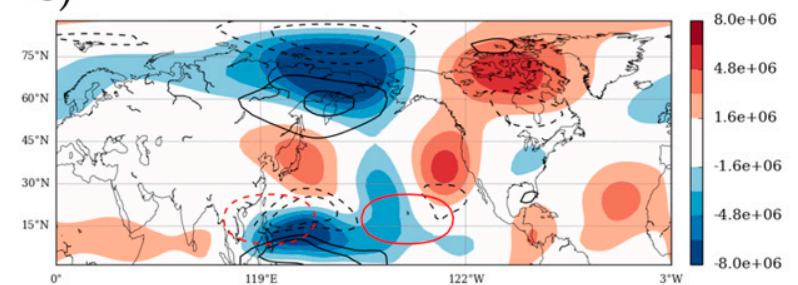

g)

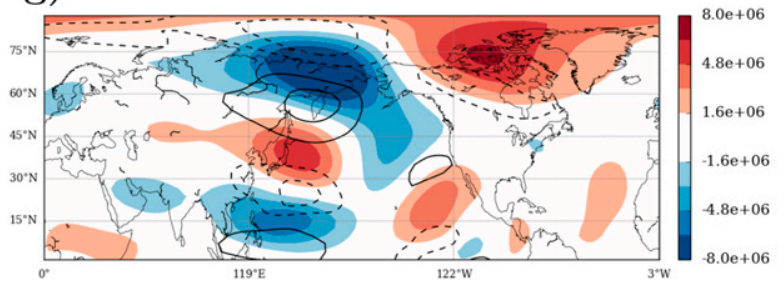

b)

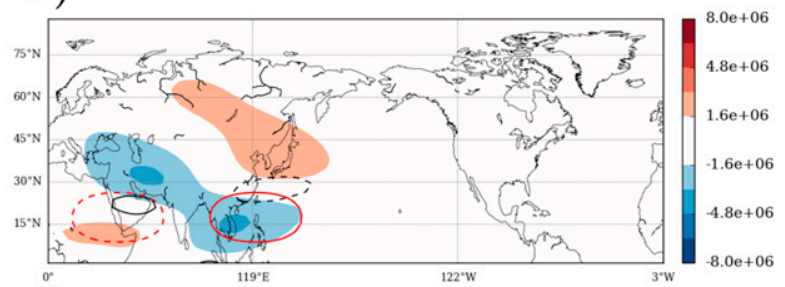

d)

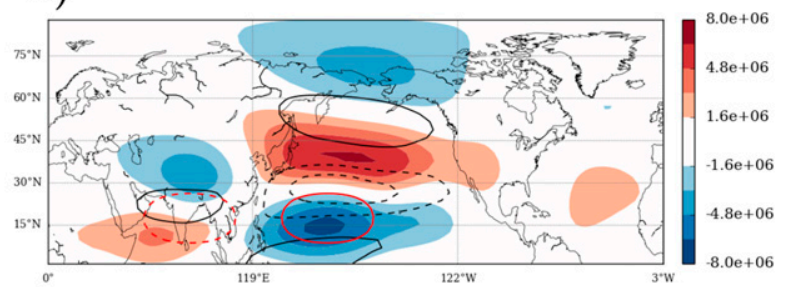

f)

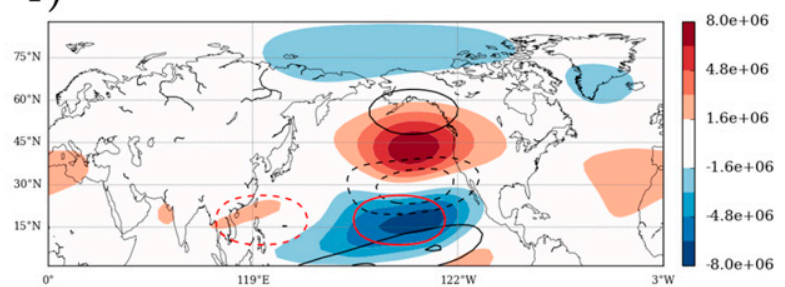

h)

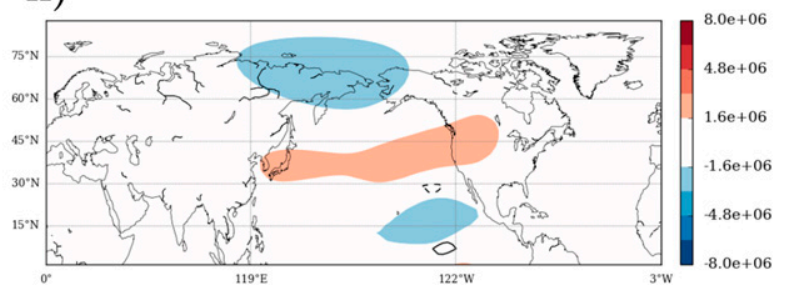

FIG. 2. Flow anomalies for the eastward-moving forcing experiment with (left) fixed and (right) time-varying reference flow. Results are represented at $t=$ (a),(b) 5, (c),(d) 15, and (e),(f) 25 days. (g),(h) Results obtained at 25 days in those experiments for which MJO forcing is turned off after 15 days. The shading corresponds to the $200-\mathrm{mb}$ streamfunction anomalies and the black contours show the 200 -mb zonal wind anomaly [contour interval (CI) is $3 \mathrm{~m} \mathrm{~s}^{-1}$; negative values are dashed; zero contour is omitted]. The MJO forcing function is represented in red contours ( $\mathrm{CI}$ is $2 \times 10^{-11} \mathrm{~s}^{-2}$; negative values are dashed) and has a maximum amplitude equal to $S_{0}=4 \times 10^{-11} \mathrm{~s}^{-2}$.

do not show the zonally elongated feature we observe at $t=15$ days and are more consistent with the results of the stationary reference flow experiments shown in Fig. 2c [see, e.g., pentad 1 in Fig. 4 of Henderson et al. (2016)]. We will come back to that point in the discussion.

In addition, at $t=25$ days, differences have also appeared over the Atlantic Ocean: in the case of the time-varying reference flow, the streamfunction anomaly consists in a ridge south of a trough (Fig. 2f). Even though the strength of this dipole seems weaker, when compared to the Pacific anomalies, than seen in the observations (see, e.g., Henderson et al. 2016), it is a clear signature of a positive NAO phase. By contrast, when the reference flow is stationary, no such clear signature is found (Fig. 2e), highlighting the importance of the flow variability in accounting for the atmospheric response to the MJO forcing. As noted above, the streamfunction anomalies in the eastern Pacific have significantly strengthened between 15 and 25 days in the time-varying reference flow case. To highlight the importance of the MJO forcing in that evolution (note that the latter has moved significantly in the Pacific basin by $t=25$ days and affects its eastern side by that time), we reproduced the experiment with the MJO forcing switched off from $t=15$ days onward. The early evolution of the atmosphere up to $t=15$ days is thus identical by design, but we found that it differs significantly at 25 days (Figs. $2 \mathrm{~g}, \mathrm{~h}$ ). This is especially true for the case of the time-varying reference flow, in which case the streamfunction anomalies have been spread away by atmospheric fluctuations, to the point that the positive-NAO-phase signature is not visible anymore. By contrast, when the background flow is stationary, 
a)

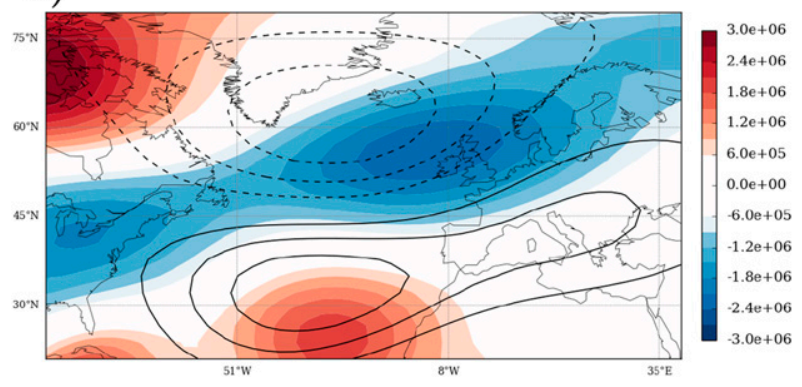

b)

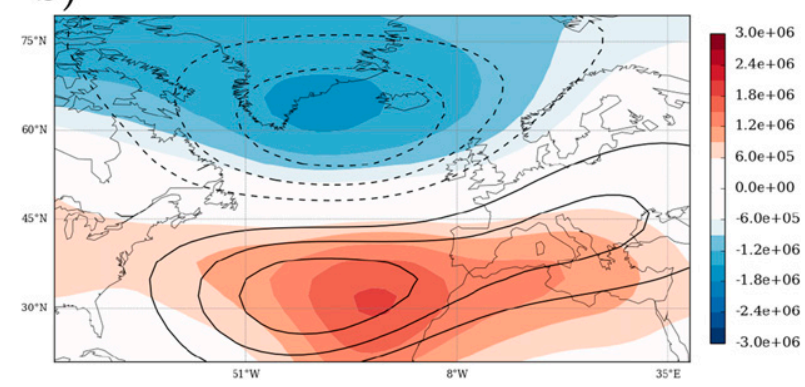

FIG. 3. Vertically averaged streamfunction anomalies (shading) and first EOF (contours) in the Atlantic region at time $t=25$ days for the case with (a) stationary and (b) time-varying reference flow. The contours correspond to $75 \%, 50 \%$, and $25 \%$ of the EOF maximum values; negative contours are dashed. Note the different shading scale compared to Fig. 2.

the anomalies remain almost constant in amplitude and display only a modest phase modification between 15 and 25 days. Taken together, these results point to the importance of both the forcing and the nature-stationary or time varying - of the reference flow in shaping the atmospheric response to the MJO. In addition, the streamfunction anomalies we find after 25 days suggest that the model is able to recover the MJO-NAO teleconnection discussed above. In the following section, we examine more quantitatively that possibility by focusing on the North Atlantic region.

\section{2) CONSEQuences fOR THE NAO}

Since the NAO is largely barotropic in nature, we concentrate on the vertically averaged streamfunction anomalies in this section. Their spatial distributions at $t=25$ days (shading) are compared with the first empirical orthogonal function (EOF; contours) in the Atlantic region in Fig. 3. The later was computed from our control simulation (see section 2 ) over the region $90^{\circ} \mathrm{W}<\lambda<20^{\circ} \mathrm{E}$ and $20^{\circ}<\phi<80^{\circ}$ using vertically averaged daily streamfunctions. Both plots confirm the results of the previous section: when the reference flow is stationary (Fig. 3a), the anomalies project poorly onto the NAO pattern, while the projection is much better for a time-varying reference flow (Fig. 3b). This can be quantified by computing the principal component (PC) value of the projection on the EOF: when normalized by the standard deviation of the PC values of the control run, it amounts to 0.07 and 0.19 for the stationary and timevarying reference flow cases, respectively. In the latter case, this value is of course an average over the 9667 short runs we performed, each of whom is characterized by its own PC value at $t=25$ days.

The PC distribution for the time-varying reference flow case is shown in Fig. 4a (red bars) and compared with an equivalent series of short runs we performed without any MJO forcing (blue bars). Both distributions display a nearly Gaussian shape with an identical standard deviation $\sigma_{\mathrm{PC}}=1.1 \times 10^{8} \mathrm{~m}^{2} \mathrm{~s}^{-1}$ and are similarly skewed toward negative values: $\gamma_{1}=-0.24$ and -0.30 for the case without and with MJO forcing, respectively. In agreement with the positive value of the PC, the red distribution features a small shift toward positive values compared to the blue distribution. Even if it is small compared to the standard deviation of the distribution, it means there are more occurrences of large, positive values of the PC following phase $3 \mathrm{MJO}$ forcing. To quantify that excess, we define the number of occurrences of the positive phase of the NAO, $N_{\mathrm{NAO}+}$, as the number of times the PC values exceeds $\sigma_{\mathrm{PC}}$ (in the following, we will refer to these events as "NAO+ days" and that threshold value as $\mathrm{PC}_{\text {crit }}$ ). For the series without any MJO forcing (i.e., the blue distribution), we find $N_{\mathrm{NAO}+}=1578$. This is close to the value we would have found $\left(N_{\mathrm{NAO}+}=1581\right)$ if the PC distribution had had a perfect Gaussian shape. For the red distribution, we find $N_{\mathrm{NAO}+}=2201$. This corresponds to an excess of NAO+ days of $39 \%$, a result comparable to that reported by Cassou (2008) for the observations. We note that an estimate of the number of $\mathrm{NAO}+$ days $\left(N_{\mathrm{NAO}+}=2193\right)$ obtained assuming a perfect Gaussian distribution biased toward positive values (with a normalized $\mathrm{PC}$ equal to 0.19 as measured above) is close to the actual figure we obtain, in agreement with the fact that the blue and red distribution have similar shapes. These results are somewhat sensitive to the exact threshold value we take to define a $\mathrm{NAO}+$ event. For example, when $\mathrm{PC}_{\text {crit }}=$ $1.5 \sigma_{\mathrm{PC}}$, we find $N_{\mathrm{NAO}+}=893$ in the presence of $\mathrm{MJO}$ heating and $N_{\mathrm{NAO}+}=563$ in its absence, that is, a relative increase of $\mathrm{NAO}+$ events by $60 \%$. When $\mathrm{PC}_{\text {crit }}=$ $2 \sigma_{\mathrm{PC}}, \mathrm{NAO}+$ events are increased by as much as $77 \%$. This would tend to indicate that extreme $\mathrm{NAO}+$ events are more sensitive to the MJO than relatively moderate events. However, the smaller number of events involved preclude any definite conclusions to be drawn here and we leave this point for further studies. 
a)

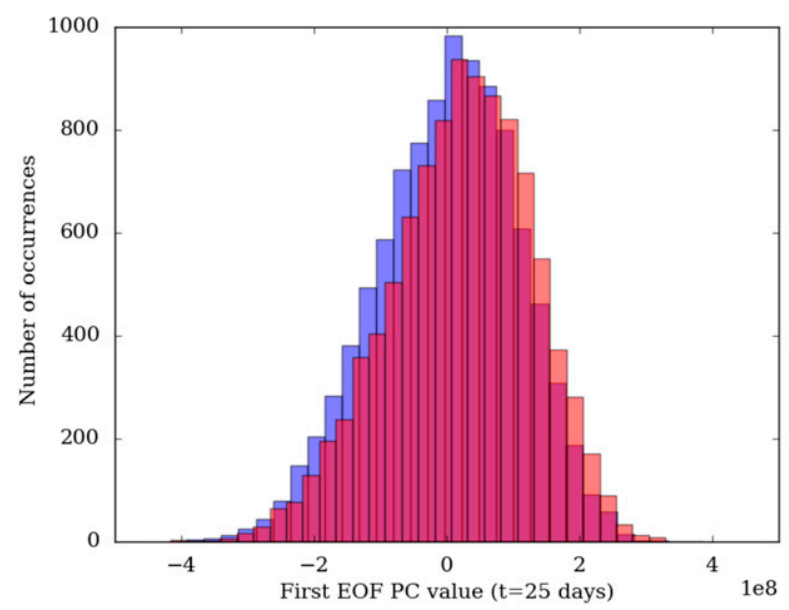

b)

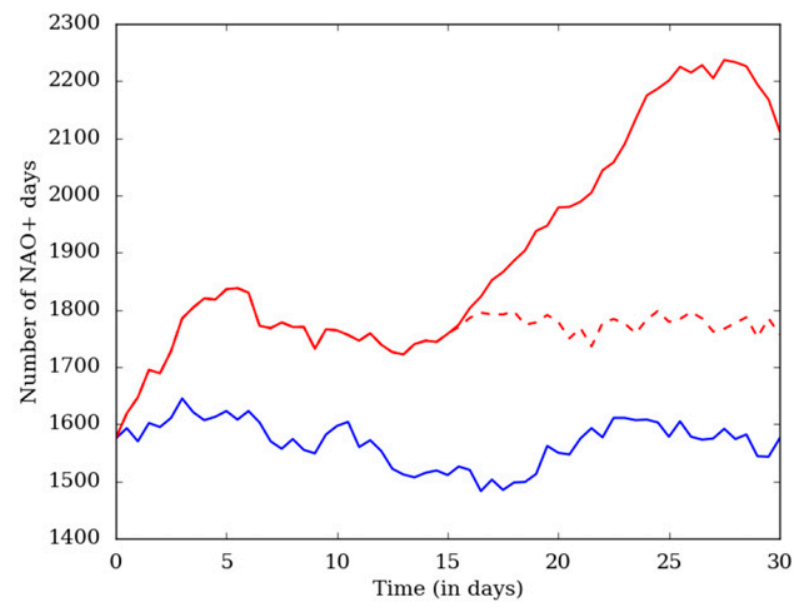

FIG. 4. (a) Probability distribution functions (PDFs) of the first EOF principal component for phase $3-$ like MJO forcing $\left(S_{0}=4 \times\right.$ $10^{-11} \mathrm{~s}^{-2}$; red) and in the absence of any MJO forcing (blue). (b) Time evolution of the number of NAO + days for phase 3 MJO forcing (red solid curve) and in the absence of any MJO forcing (blue solid curve). The dashed red curve corresponds to the case for which the MJO forcing is switched off at $t=15$ days.

The analysis presented above can be reproduced at different times after MJO forcing is switched on to evaluate the time evolution of the number of $\mathrm{NAO}+$ days. This is done in Fig. 4b (red curve) where it is compared with the case without MJO forcing (blue curve), using $\mathrm{PC}_{\text {crit }}=\sigma_{\mathrm{PC}}$. As expected, the blue curve is flat: the different times of the simulations are all statistically equivalent. The situation is different with MJO forcing: the number of $\mathrm{NAO}+$ days first increases to about 1750 days (compared to the unperturbed value of about 1600 days) during the first few days of the experiments and then remains roughly constant for about 15 days. An almost linear increase with time up to $N_{\mathrm{NAO}+} \sim 2200$ follows until $t=25-27$ days. In the experiment in which we turned the MJO forcing off at $t=15$ days (red dashed curve), this increase is not observed and the number of $\mathrm{NAO}+$ days remains roughly constant from $t=5$ days until the end of the run, in agreement with the bottom panel of Fig. 2.

\section{b. Fixed MJO forcing}

When using a fixed MJO forcing (Fig. 5), the flow anomalies that develop have strong similarities with the features described above: a large-scale stationary Rossby wave develops in a few days and propagates eastward regardless of the nature (fixed or time varying) of the reference flow. There are some differences, though: first, the flow response for the fixed MJO forcing case tends to be stronger than that for the previously described eastward-moving MJO forcing, and particularly so in the western Pacific area. This is due to the fact that the forcing always has the same phase and reinforces existing anomalies during the entire run. Second, when the background flow is time varying, the ridge south of Alaska is weaker than observed previously at $t=$ 25 days, reflecting the absence of the forcing in the eastern Pacific area at that time. But despite these differences, we recover the main features described above for the case of an eastward-moving MJO forcing: as before, the streamfunction anomalies in the Pacific region are more zonally elongated when the reference flow is time varying. At $t=25$ days, we also recover a dipolar structure typical of the positive phase of the NAO in the Atlantic, while no such a feature is seen either for the stationary reference flow case or when the forcing is turned off at $t=15$ days. Performing the same analysis as for the case of the eastward-moving MJO forcing (not shown), we found $N_{\mathrm{NAO}+}=2200$ at $t=25$ days, indicating a similar positive bias of the NAO regardless of whether the MJO forcing is fixed or eastward moving.

\section{c. Summary}

The results we obtained are conveniently summarized with the help of Fig. 6, in which we show the time evolution of the projection of the vertically averaged streamfunction onto the first EOF for the eight simulations we performed. Qualitatively, we observe the same evolution with time, regardless of whether the MJO forcing is moving eastward (Fig. 6a) or fixed (Fig. 6b): The PC first grows at early times and shows a first peak at $t=5$ days, independently of the nature of the background flow (time varying or stationary). As will become clearer in the following section, this first peak is due to a mix of a remote residual influence of the forcing in the Atlantic and of early westward Rossby wave propagation from the Indian 
a)

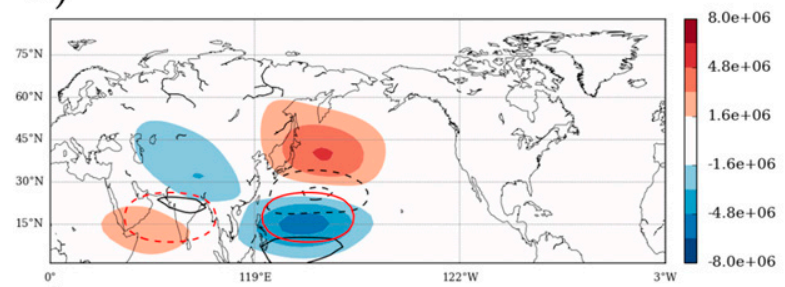

c)

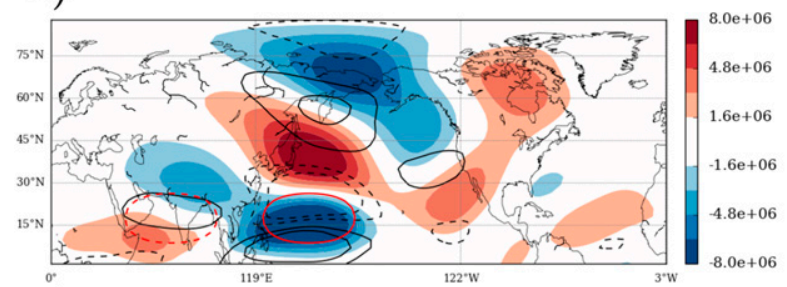

e)

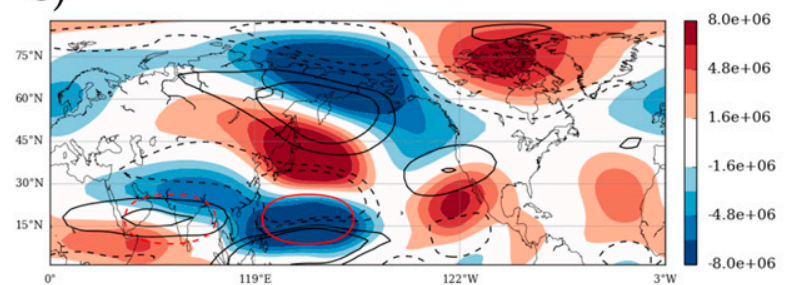

g)

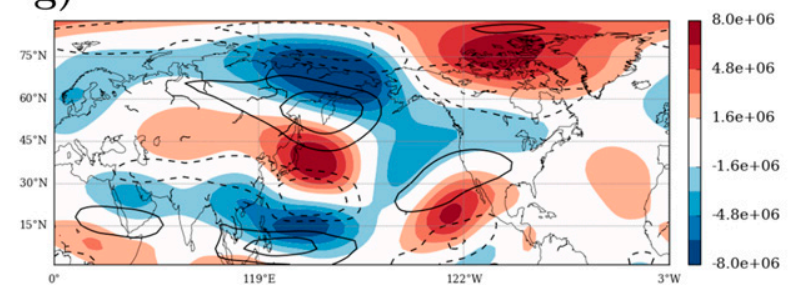

b)

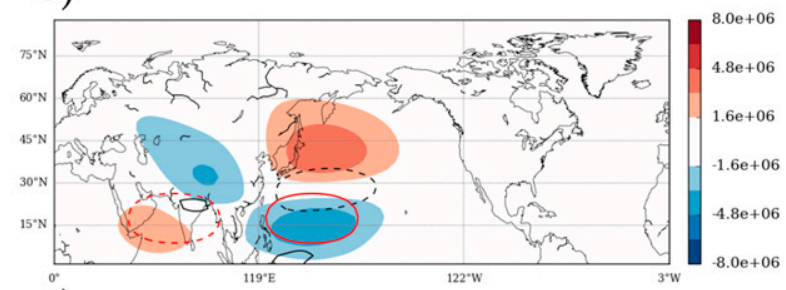

d)

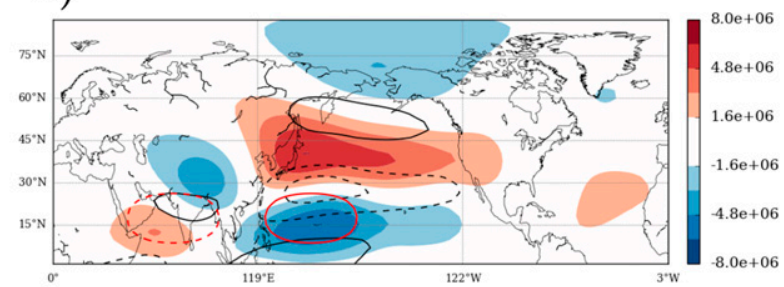

f)

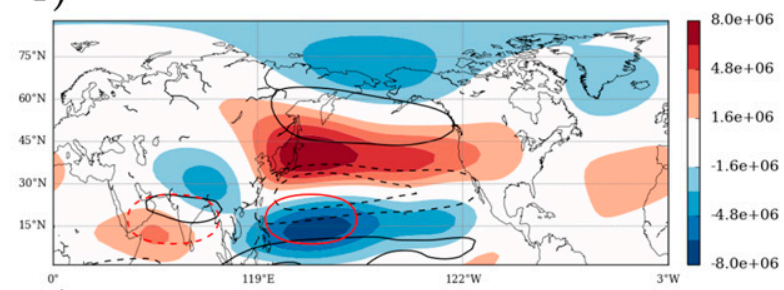

h)

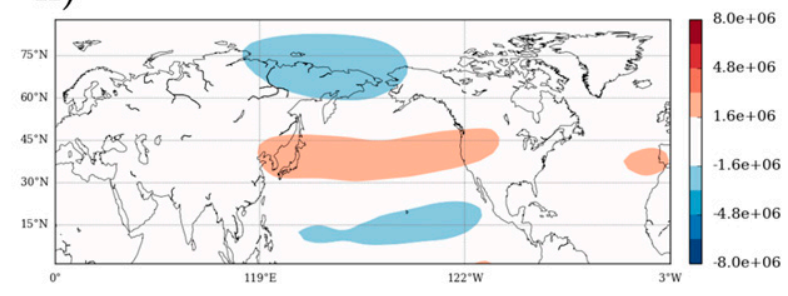

FIG. 5. As in Fig. 2, but for the case of a fixed MJO forcing.

Ocean, as described for example by Lin and Brunet (2018) for the case of the MJO or by Lin and Wu (2012) for the case of the Indian monsoon. We stress that the PC values that are reached at that time remain moderate and cannot explain the amplitude of the observed bias of the NAO that follows MJO phase 3 since it is only of order $10 \%$ (see Fig. 4b), that is, smaller than the $30 \%$ typical biases reported by Cassou (2008) in the observations.

At $t>5$ days, the model results depend on the nature of the reference flow. When it is stationary, the PC values oscillate around zero regardless of the nature of the forcing (blue solid lines). Further analysis (not shown) suggests that this is due to phase mixing of Rossby waves of different wavelengths and phase speeds. The decrease observed between $t=5$ and 15 days is due to the eastwardpropagating Rossby wave triggered by the MJO forcing that projects negatively on the NAO when it reaches the Atlantic area, while the increase between $t=15$ and 25 days is associated with an eastward-propagating
Rossby wave of higher wavenumber with an equivalent barotropic structure. The latter creates higher wavenumber streamfunction anomalies in the Pacific and enhances the ridge in the southern part of the North Atlantic area such that it projects positively on the NAO (see Fig. 2e). By contrast, for time-varying reference flows, the PC values remain roughly constant until $t \sim 15$ days before growing to reach values compatible with the observations at $t=25$ days (red solid lines). We investigate the origin of that increase in the following section. For all cases, the PC values are smaller in those experiments for which we turn off the forcing at $t>15$ days (dashed lines). Such a fast modification so far away from the location of the MJO might seem surprising. We will come back to that issue in the following section and show that this is due to a remnant forcing over western Europe.

Taken together, we conclude that our model simulations are able to capture the lagged correlation between the $\mathrm{MJO}$ and the NAO and highlight the importance of both 
a)

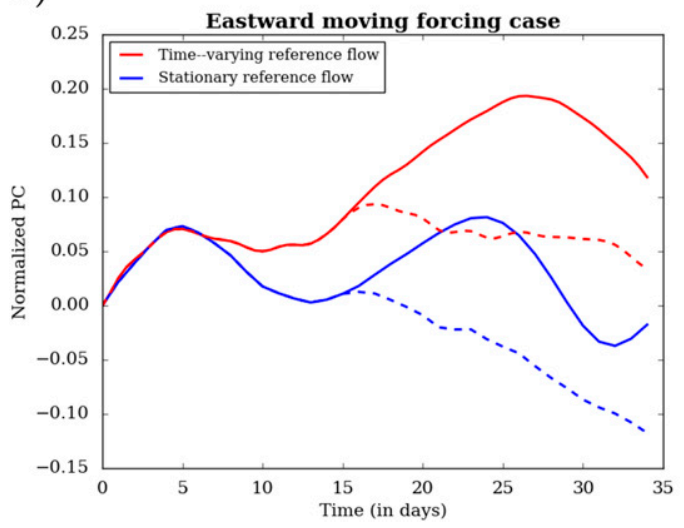

b)

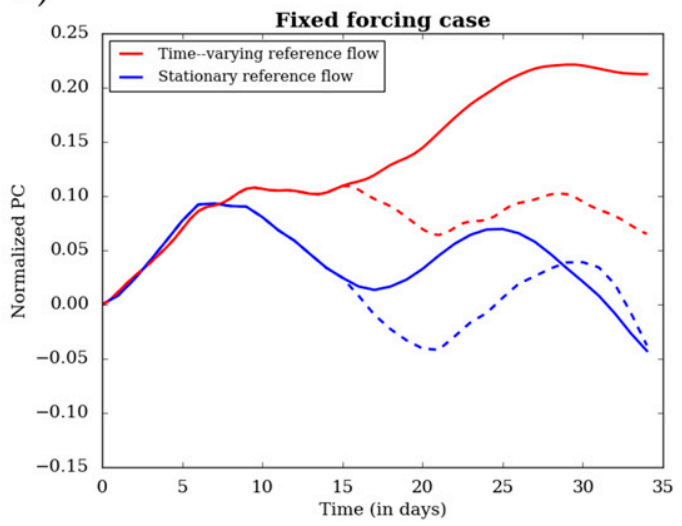

FIG. 6. Time evolution of the normalized PC1 value for the case of (a) an eastward-moving MJO forcing and (b) a fixed MJO forcing. The red and blue curves correspond to the case of time-varying and stationary reference flow, respectively. Dashed curves are the results for those cases for which the forcing is turned off after 15 days.

stationary Rossby waves and the midlatitude atmospheric variability in shaping the North Atlantic response to the MJO. We now turn to their physical interpretation.

\section{Interpretation and physical mechanisms}

In this section, we address the following questions: what makes the streamfunction anomalies zonally elongated in the Pacific region at $t=15$ days? What drives the growth of the positive NAO phase between $t=15$ and 25 days? This is done by means of a budget analysis of the total streamfunction $\psi=\psi_{1}+\psi_{2}+\psi_{3}$ that we successively apply to the Pacific area between $t=5$ and 15 days and to the Atlantic area between $t=15$ and 25 days.

\section{a. Method}

Summing the governing equations over the three model levels (neglecting the hyperdiffusion terms), we obtain a tendency equation for the vertically integrated streamfunction $\psi=\psi_{1}+\psi_{2}+\psi_{3}$ :

$$
\frac{\partial \psi}{\partial t}=\nabla^{-2} \sum_{i=1}^{3} J\left(q_{i}, \psi_{i}\right)+\nabla^{-2} S-\frac{\psi_{3}}{\tau_{E}}
$$

where $S=S_{1}+S_{2}+S_{3}$ is the vertically integrated total forcing and $\nabla^{-2}$ represents the inverse of the Laplacian operator.

As was done above, let us now decompose any given variable into the sum of its time-mean, low-frequency, and high-frequency components. ${ }^{2}$ For example, $q_{i}$ is written as

\footnotetext{
${ }^{2}$ These data were computed, as before, using a Lanczos filter with an 8-day cutoff period. At any given time, data are thus required from 4 days in the past to 4 days in the future. As a result, we can only calculate filtered data from $t=4$ to $t=30$ days.
}

$$
q_{i}=\bar{q}_{i}^{\mathrm{REF}}+q_{i}^{L}+q_{i}^{H} .
$$

In the analysis that follows, we calculate ${\overline{q_{i}}}^{\mathrm{REF}}$ from a control run that lacks any MJO forcing. In that case, both $q_{i}^{L}$ and $q_{i}^{H}$ have vanishing time-averaged values. However, in the presence of a nonzero MJO forcing, this is not the case anymore for $q_{i}^{L}$, while it remains true for $q_{i}^{H}$.

Plugging such a decomposition for $q_{i}$ and $\psi_{i}$ into Eq. (15), we obtain

$$
\frac{\partial \psi}{\partial t}=\sum_{\alpha=0}^{5} \xi_{\alpha}+\nabla^{-2} S-\frac{\psi_{3}}{\tau_{E}},
$$

where $\xi_{\alpha}$ are defined by the following relations:

$$
\begin{aligned}
& \xi_{0}=\nabla^{-2} \sum_{i=1}^{3} J\left(\bar{q}_{i}^{\mathrm{REF}}, \bar{\psi}_{i}^{\mathrm{REF}}\right), \\
& \xi_{1}=\nabla^{-2} \sum_{i=1}^{3}\left[J\left(\bar{q}_{i}^{\mathrm{REF}}, \psi_{i}^{L}\right)+J\left(q_{i}^{L}, \bar{\psi}_{i}^{\mathrm{REF}}\right)\right], \\
& \xi_{2}=\nabla^{-2} \sum_{i=1}^{3} J\left(q_{i}^{L}, \psi_{i}^{L}\right), \\
& \xi_{3}=\nabla^{-2} \sum_{i=1}^{3} J\left(q_{i}^{H}, \psi_{i}^{H}\right), \\
& \xi_{4}=\nabla^{-2} \sum_{i=1}^{3}\left[J\left(\bar{q}_{i}^{\mathrm{REF}}, \psi_{i}^{H}\right)+J\left(q_{i}^{H}, \bar{\psi}_{i}^{\mathrm{REF}}\right)\right], \\
& \xi_{5}=\nabla^{-2} \sum_{i=1}^{3}\left[J\left(q_{i}^{L}, \psi_{i}^{H}\right)+J\left(q_{i}^{H}, \psi_{i}^{L}\right)\right] .
\end{aligned}
$$

The physical meaning of these different terms is as follows: $\xi_{0}$ describes the advection of the mean PV by the mean flow; $\xi_{1}$ and $\xi_{4}$ encapsulate the linear evolution of the low- and high-frequency anomalies, respectively; 
$\xi_{2}$ and $\xi_{3}$ result from the nonlinear interaction among the low- and high-frequency anomalies, respectively; and finally $\xi_{5}$ evaluates the nonlinear coupling between low- and high-frequency fluctuations. For the series of runs with a time-varying reference flow, we further make an ensemble average of each term over the entire 9967 members of the two ensembles, one with MJO forcing and one without MJO forcing, and subtract the second from the first. We note $\delta \xi_{\alpha}$ the functions thus obtained, which depend on space and time. Likewise, the streamfunction anomalies $\delta \psi$ are computed from the two ensembles. It is clear that $\delta \xi_{0}=0$. Also $\delta \xi_{4}$ should exactly vanish, both in the stationary reference flow experiments (because of the absence of any synoptic activity over the duration of the runs in that case) and in an ensemble-averaged sense for the series of time-varying reference flow experiments. In practice, we indeed found that $\delta \xi_{4}$ is very small compared to the other terms. Likewise, $\delta \xi_{5}$ is never dominant and tends to reinforce $\delta \xi_{3}$ in general. We will ignore the former in our analysis below. However, our results indicate that $\delta \xi_{1}, \delta \xi_{2}$ and $\delta \xi_{3}$ all contribute significantly to the streamfunction budget. No further simplification is thus possible, but we found it convenient to group all the linear terms together and define

$$
\delta \xi_{1}^{\mathrm{Lin}}=\delta \xi_{1}-\frac{\delta \psi_{3}^{L}}{\tau_{E}}
$$

The overall anomalous streamfunction budget can finally be formally written as

$$
\frac{\partial \delta \psi}{\partial t}=\delta \xi_{1}^{\mathrm{Lin}}+\delta \xi_{2}+\delta \xi_{3}+\delta \xi_{5}+\delta F+R,
$$

where $\delta F=\nabla^{-2} S^{\mathrm{MJO}}$ will be referred to as the forcing term and $R$ is a residual that includes the horizontal hyperdiffusion and all the terms that do not exactly average to zero for numerical reasons (e.g., $\delta \xi_{4}$ ).

\section{b. Budget analysis in the Pacific region}

We first focus on the streamfunction budget between 5 and 15 days over the Pacific region and contrast the results obtained when the reference flow is time varying (Fig. 7) and stationary (Fig. 8). In both cases, we first compare the sum of Eq. (19) right-hand-side terms (except the residual $R$ ), time averaged over the interval of interest, with a direct estimate of the streamfunction tendency calculated using the models outputs at times $t=5$ and 15 days. We find a good agreement between the two methods (Figs. 7a and 8a), which demonstrates that the streamfunction budget we perform is closed and that the residual $R$ is much smaller than the other terms. This validates our approach.
In the budget, the sum of the linear and forcing terms (Figs. $7 b$ and $8 b$ ) is similar for both cases, even if its amplitude tends to be slightly smaller when the reference flow is time varying. For the stationary reference flow case, it accounts for most of the total streamfunction anomaly tendency (note the similarity between Figs. 8a and 8b): as discussed above and in agreement with previous results, the flow response is mostly linear and takes the form of a barotropic stationary Rossby wave. Accordingly, both the low- and high-frequency terms are very small in that case (Figs. 8c,d) and do not contribute to the overall streamfunction budget. This is not the case for the time-varying reference flow case, for which both $\delta \xi_{2}$ and $\delta \xi_{3}$ take significant values (Figs. 7c,d). $\delta \xi_{2}$ is essentially the opposite of the linear and forcing terms, and thus acts as a form of drag that moderates the linear response of the atmosphere to the MJO forcing. In addition to a small response over India and Pakistan, $\delta \xi_{3}$ mainly consists in a dipole over the Pacific area with negative values in the subtropical region and positive values extending over the whole Pacific from the Korean Peninsula to California. This dipolar structure of $\delta \xi_{3}$ largely explains the zonally elongated structure of the total streamfunction anomaly. It reflects the northward shift of the eastern Pacific storm track observed at $t=15$ days, which can be quantified using the high-frequency eddy kinetic energy [EKE; defined as $\left.\left[\left(u_{i}^{H}\right)^{2}+\left(v_{i}^{H}\right)^{2}\right] / 2\right\}$. Its $200-\mathrm{mb}$ anomalies feature a strong dipolar structure in the eastern Pacific basin (Fig. 9a), which is the signature of the poleward-shifted Pacific storm track. It is also associated with synoptic eddies propagating more equatorward, as measured using the $\mathbf{E}$ vector introduced by Trenberth (1986). The E-vector divergence that results is well correlated with the zonal wind anomalies in the eastern Pacific (Fig. 9b).

To conclude, the picture that emerges from this analysis is that the western Pacific area flow modifications can be attributed to the MJO atmospheric linear response while the eastern Pacific anomalies are a consequence of a modified transient eddy activity that ensues. This is consistent with the analysis of Sakaeda and Roundy (2014), who reported a two-way interaction between transients eddies and the intraseasonal 200-mb zonal wind in the Pacific.

\section{c. Budget analysis in the Atlantic region}

We next analyze the streamfunction budget between $t=15$ and $t=25$ days in the northern Atlantic region.

For the stationary reference flow case (not shown), the streamfunction tendency over that period has a spatial structure similar to the vertically integrated streamfunction itself at $t=25$ days such as represented 
a)

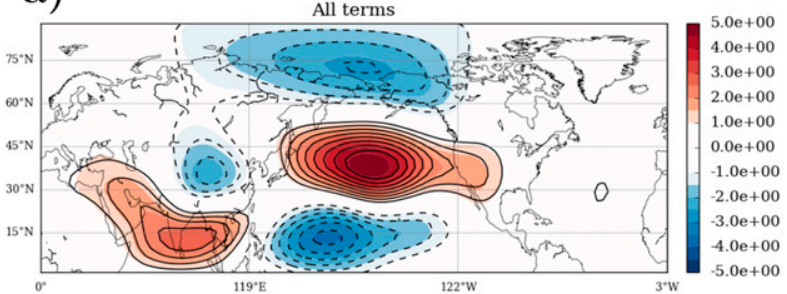

c)

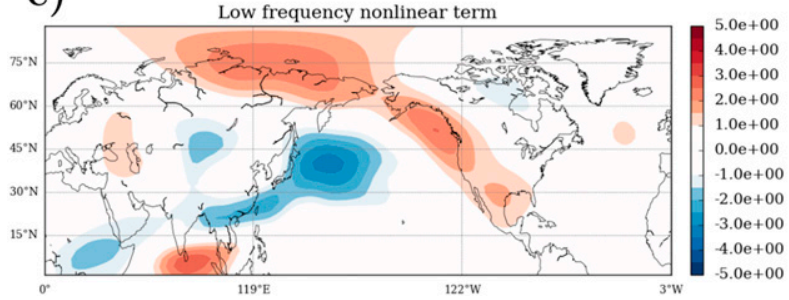

b)

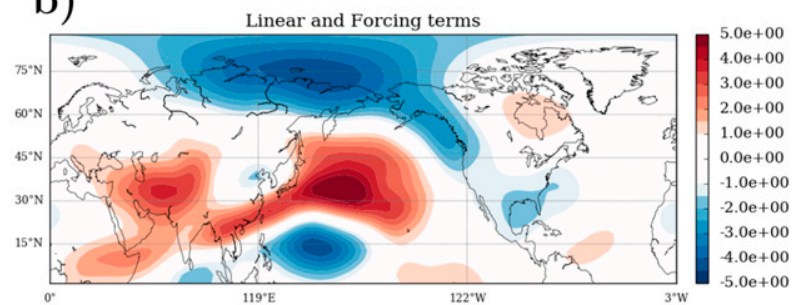

d)

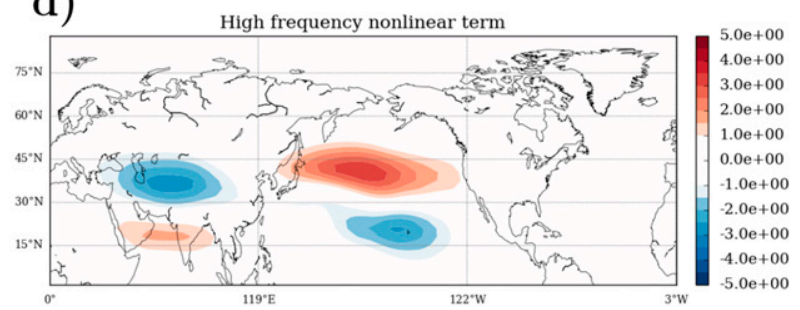

FIG. 7. Streamfunction budget analysis between $t=5$ and 15 days performed according to Eq. (19) for the case of an eastward-moving MJO forcing and a time-varying reference flow. Shading represents (a) the sum of all terms except $R$, (b) $\delta \xi_{1}^{\mathrm{Lin}}+\delta F$, and (c) $\delta \xi_{2}$, and (d) $\delta \xi_{3}$. In (a), contours show $\partial \delta \psi / \partial t$ evaluated directly from the model streamfunction (CI is identical to the shading interval).

in Fig. 3. It is almost completely accounted for by the linear and forcing terms, with $\delta \xi_{2}$ and $\delta \xi_{3}$ being negligible over the region of interest. This is similar to the results discussed above for the Pacific region at earlier time.

For the time-varying reference flow case, we again find a closed budget (Fig. 10a) with significant contributions from the nonlinear terms. For a simpler interpretation of the results, contours of the first EOF are overlaid on the representation of the different tendency terms in the other panels (Figs. 10b-d). The linear and forcing terms differ from the stationary reference flow case (cf. Figs. 10b and 3a). This is because the altered anomalies in the Pacific area modify the stationary Rossby wave propagation to the Atlantic. Its projection on the first EOF is poor in the eastern and central Atlantic area but becomes better over western Europe. As was the case for the Pacific area, we find that $\delta \xi_{2}$ is essentially the opposite of $\delta \xi_{1}^{\mathrm{Lin}}+\delta F$ (Fig. 10c). This is particularly true in the eastern Atlantic and over the North American continent, where it projects positively on the first EOF, while this is not so much the case over western Europe. Finally, we find that the largest projection on the first EOF comes from the high-frequency nonlinear term (Fig. 10d): we thus conclude that the growth of the NAO+ between 15 and 25 days is due to the modification of the Atlantic jet synoptic activity, which is perhaps the most a)

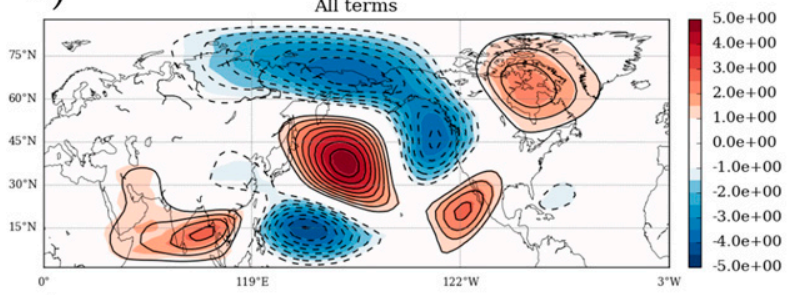

c)

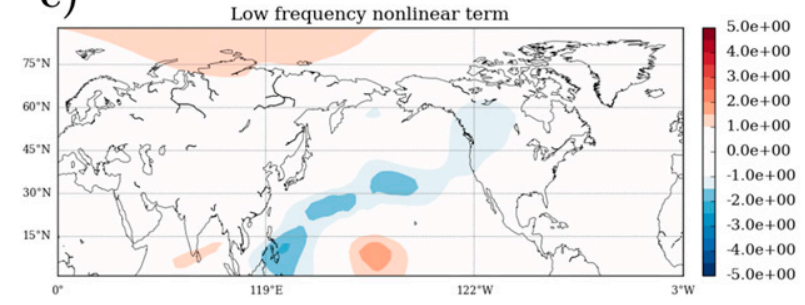

b)

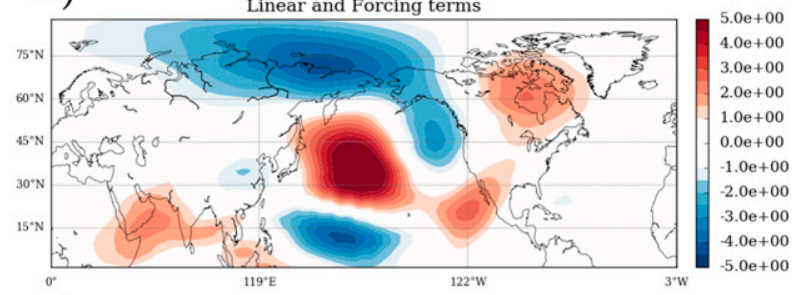

d)

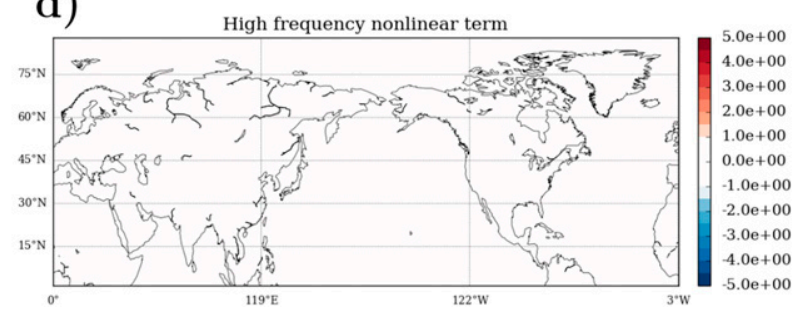

FIG. 8. As in Fig. 7, but for the case of a stationary reference flow. 
a)

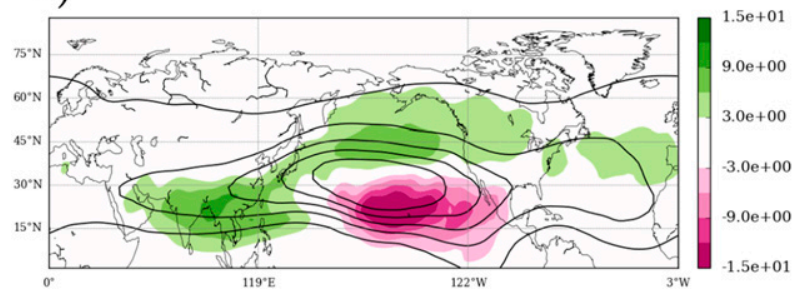

b)

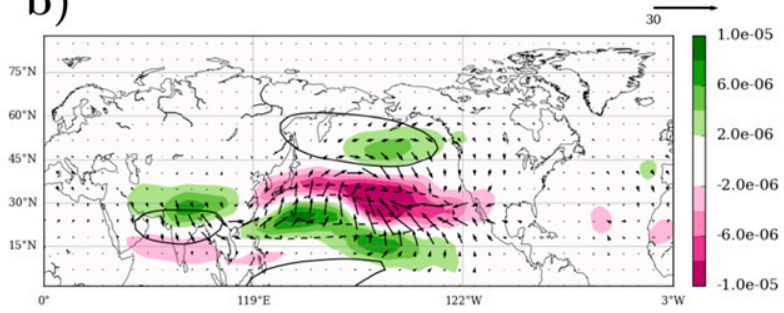

FIG. 9. (a) Ensemble average of the high-pass-filtered 200-mb EKE anomaly (shading; $\left.\mathrm{m}^{2} \mathrm{~s}^{-2}\right)$ for phase $3 \mathrm{MJO}$ forcing $\left(S_{0}=4 \times 10^{-11} \mathrm{~s}^{-2}\right)$ at $t=15$ days. The black contours represents the climatological EKE (CI: $20 \mathrm{~m}^{2} \cdot \mathrm{s}^{-2}$ ). (b) Ensemble average of the $\mathbf{E}$ vector (arrows) and divergence (shading; $\mathrm{m} \mathrm{s}^{-2}$ ) anomalies for phase $3 \mathrm{MJO}$ forcing $\left(S_{0}=4 \times 10^{-11} \mathrm{~s}^{-2}\right)$. The black contours represent the zonal wind anomalies.

important result of our paper. Again, the consequence of this modified synoptic activity also manifests itself as a northward shift of the Atlantic storm track at $t=25$ days (Fig. 11a) along with an anomalous equatorward propagation of synoptic eddies that results in an increased $\mathbf{E}$-vector divergence in the northern Atlantic basin, shifting the 200-mb jet poleward (Fig. 11b).

How robust are these results to the exact nature of the MJO forcing? In Fig. 12, we compare the time evolution of the projections of the different terms onto the first EOF for the eastward-moving and fixed MJO forcing cases. In contrast with the previous figures, we also separate the contributions arising from the linear and forcing terms, because the latter can have a nonnegligible contribution in some circumstances. It might be surprising at first glance to find such a nonvanishing contribution of the forcing so far away from the actual location of the MJO. However, since we use a nonzero vertically integrated forcing in $\mathrm{PV}$, its contribution to the streamfunction budget - defined through an inverse Laplacian - is nonlocal and affects the entire globe. For the eastward-moving MJO forcing case, the forcing projection onto the first EOF is the largest contribution to the streamfunction budget early in the simulations $(t<5$ days $)$ and is dominated by a nonvanishing contribution over western Europe (not shown). This creates an instantaneous response in the Atlantic, explaining why all nonlinear terms in the streamfunction budget differ from zero: $\delta \xi_{2}$ and $\delta \xi_{3}$ are found to cancel each other, $\delta \xi_{5}$ is negligible. The value of $\delta \xi_{1}^{\mathrm{Lin}}$ is small and positive: it is composed a)

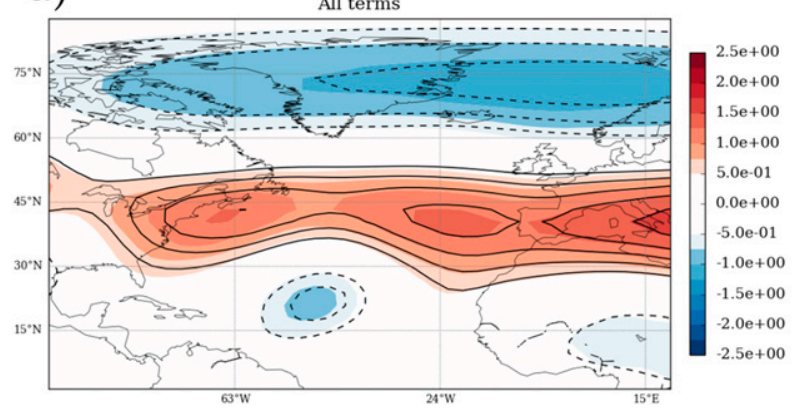

c)

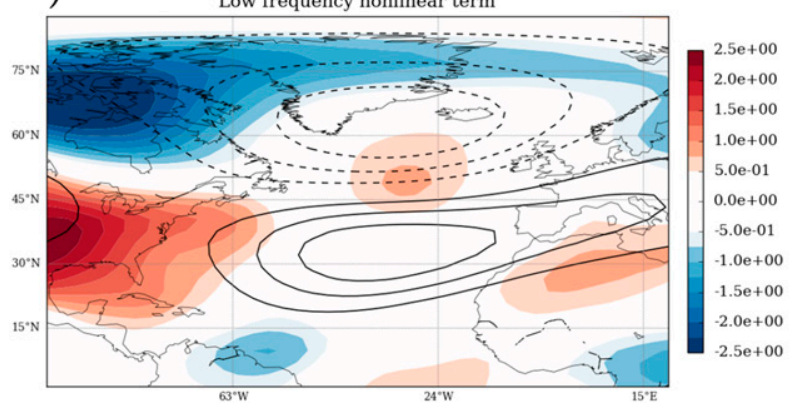

b)

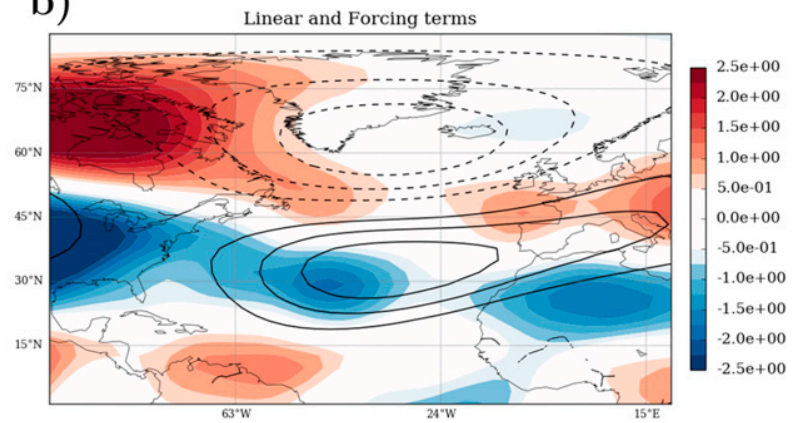

d)

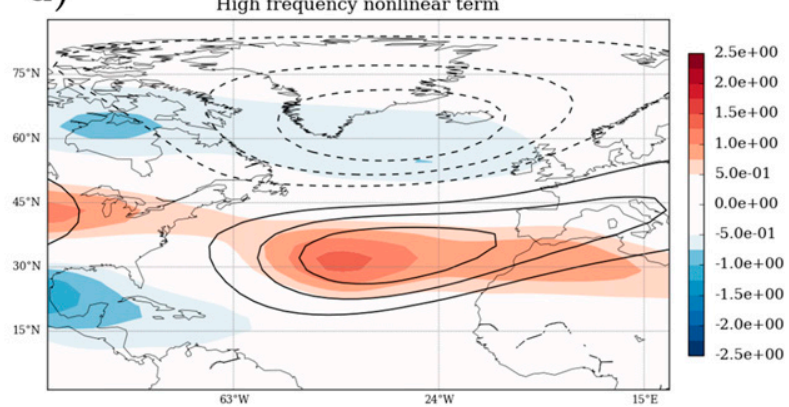

FIG. 10. As in Fig. 7, but for the streamfunction budget between 15 and 25 days and focusing on the northern Atlantic basin. (b)-(d) The contours represent the first EOF in that region. Contours are plotted at $25 \%, 50 \%$, and $75 \%$ of the first EOF extrema; negative contours are dashed. 
a)

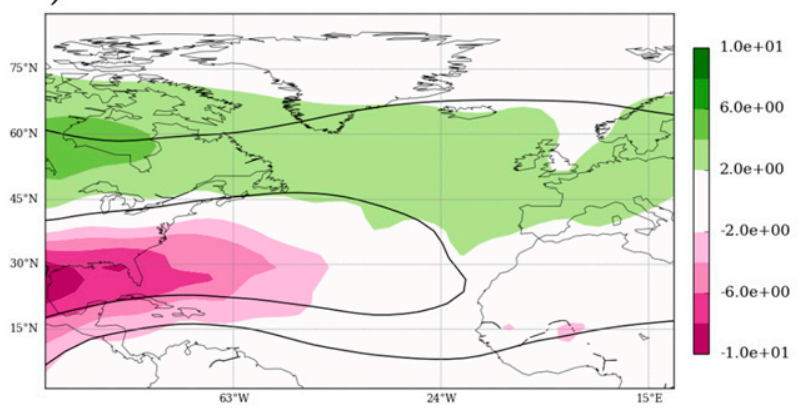

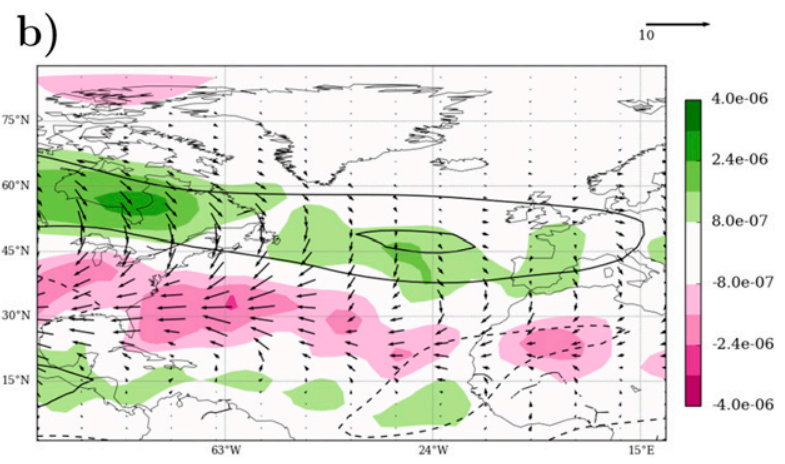

FIG. 11. As in Fig. 9, but at time $t=25$ days and focusing on the northern Atlantic basin.

of a positive contribution in the central Atlantic area and a negative contribution over western Europe (not shown). Overall, the total projection is approximately given by the sum of the linear and forcing term (solid blue curve in Fig. 12a) and is positive. This explains the early bump at $t=5$ days we described in Fig. 6a. The negligible values of the nonlinear terms explain why the same feature is obtained in the stationary reference flow case (dashed line in Fig. 6a). At times $t>5$ days, the projection of $\delta F$ remains positive but steadily decreases as the MJO moves eastward in the Pacific area. Its impact on the overall budget becomes less and less important: between $t=15$ and $t=25$ days, it amounts to about $9.5 \%$ of the sum of all positive terms. By contrast, the nonlinear terms $\delta \xi_{2}$ and $\delta \xi_{3}$ respectively contribute to $28 \%$ and $52 \%$ of all the positive terms and account for most of the increase of the mean PC observed over that period. This highlights the importance of the nonlinear interactions between transient eddies in promoting the positive phase of the NAO.

A similar picture emerges for the fixed MJO forcing case (Fig. 12b), showing that these results are robust to the exact characteristics of $S_{i}^{\mathrm{MJO}}$. The forcing term is positive at all times as above but with a smaller and constant amplitude due to the more eastern position of the MJO in the fixed forcing experiment at early times ( $t<5$ days) compared to the eastward-moving forcing experiment considered above. At $t<5$ days, as before, the total projection is positive and is mostly due the sum of the linear and forcing terms. As for the case of an eastward-moving MJO forcing, this explains the first peak of both the solid and dashed lines in Fig. $6 \mathrm{~b}$. However, in contrast to the previous case, the linear terms completely dominate over the forcing terms and account for most of the total response. As discussed by Lin and Brunet (2018), this is due to the westward propagation of a Rossby wave at early times (see Fig. 13). Our findings confirm their analysis. However, the anomalies it creates in the Atlantic area is too small to explain the $\mathrm{NAO}+$ excess alone and reduces for $t>5$ days as a consequence of the eastward-propagating Rossby wave arriving in the Atlantic (see section 3). At times $t>$ 10 days, the sum of the linear and forcing term becomes negative, while the low- and high-frequency nonlinear a)

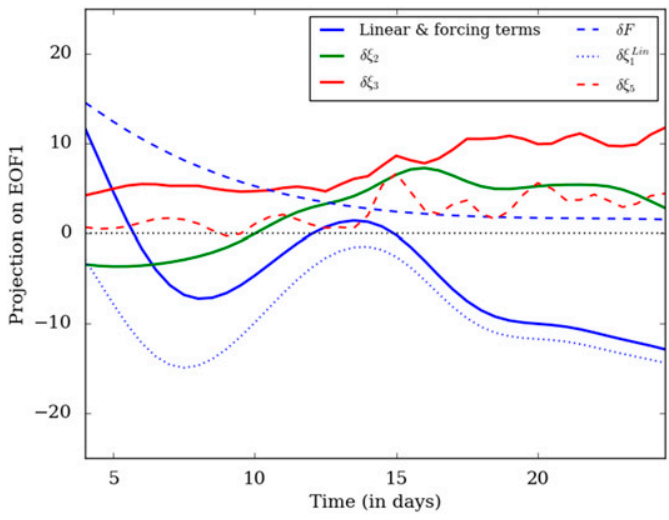

b)

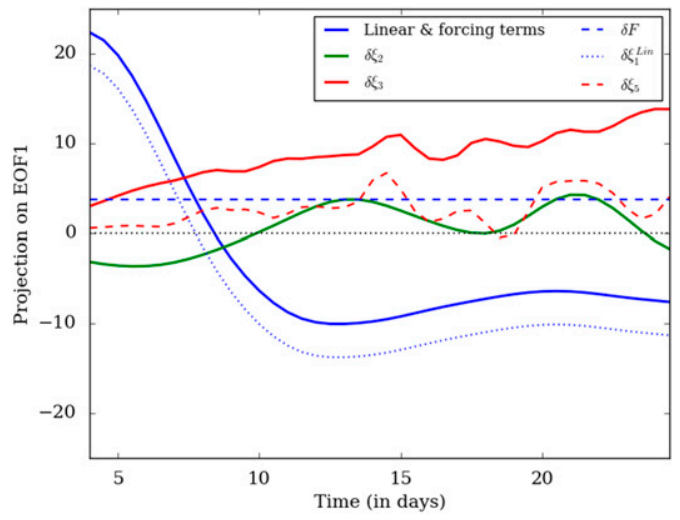

FIG. 12. Time evolution of the projection of the different terms appearing in the vorticity budget analysis on the first EOF in the Atlantic region for (a) an eastward-moving and (b) a fixed MJO forcing. 
a)

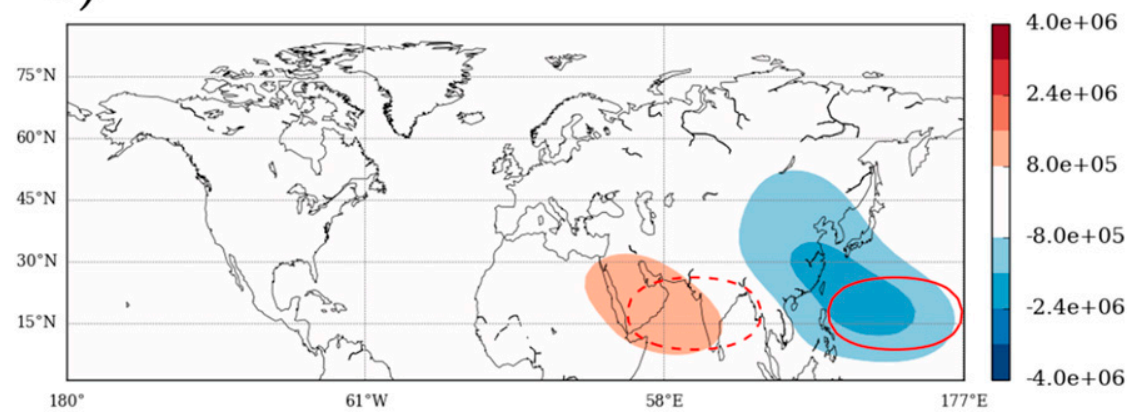

b)

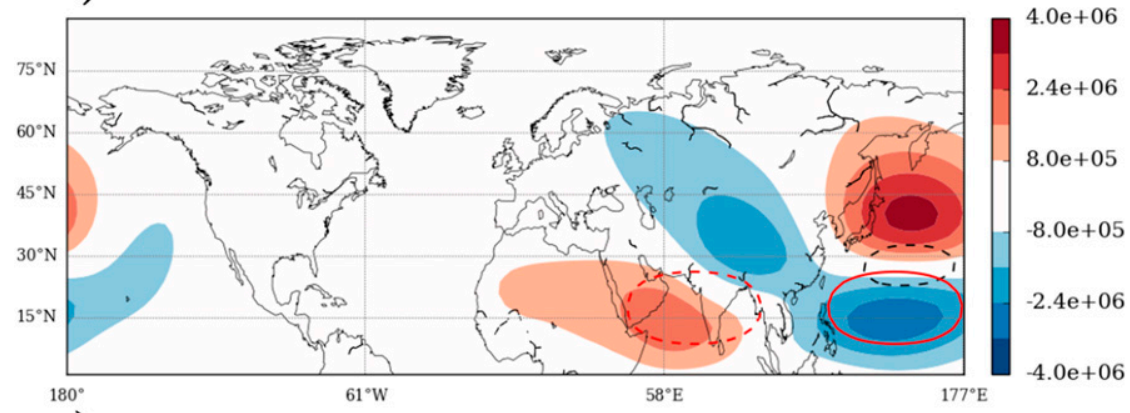

c)

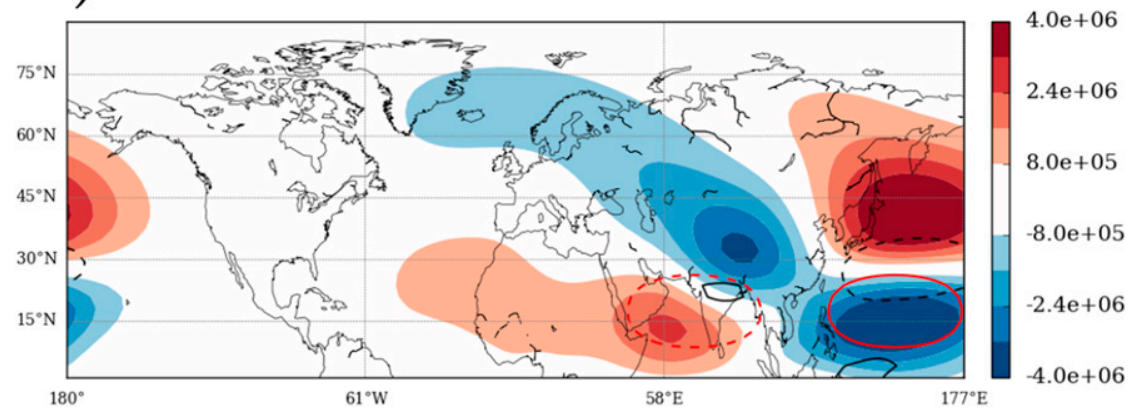

FIG. 13. The 200-mb streamfunction anomalies for the constant MJO forcing experiment $\left(S_{0}=4 \times 10^{-11} \mathrm{~s}^{-2}\right)$ and a time-varying reference flow at time $t=$ (a) 1 , (b) 3, and (c) 5 days. Note the different color scales and the different coordinates compared to Fig. 5.

terms are positive, which again highlights the important role of nonlinear transient eddies at later times.

The previous discussion also helps understand the fast response of the flow over the Atlantic to turning the MJO forcing off at $t=15$ days. As described above, $\delta F$ dominates the streamfunction budget over western Europe. As such, it has an important influence on the Rossby waves that propagate over the Atlantic region. In those experiments for which it is suppressed at $t=15$ days, the time evolution of the different components entering the streamfunction budget shows that $\delta \xi_{1}^{\text {lin }}$ indeed decreases in just a few days (not shown) and explains the decrease of the PCs observed in those cases. This decrease essentially mirrors the early bump seen in Fig. 6. We thus conclude that it is due to the suppression of the westward-propagating Rossby wave described above. Of course, such a fast response is due to the nature of the MJO forcing we use and we would expect it to be delayed if we were using a more realistic setup.

\section{Sensitivity studies}

To demonstrate the robustness of our results, we now present a series of sensitivity tests in which we vary the sign and the amplitude of the eastward MJO forcing.

\section{a. Phase 6 MJO forcing}

As described in the introduction, Cassou (2008) also found an excess of the negative phase of the NAO 
a)

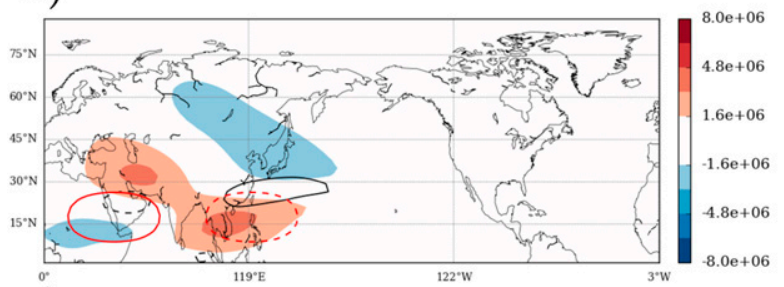

c)

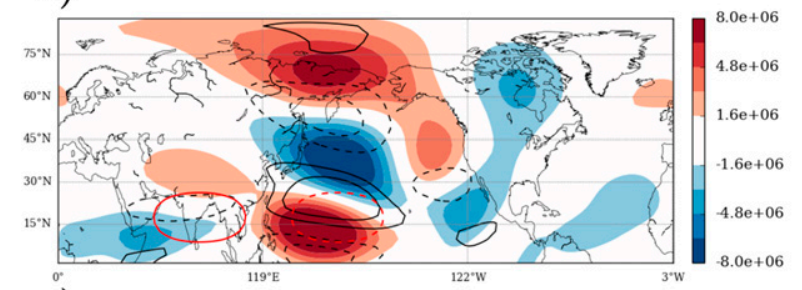

e)

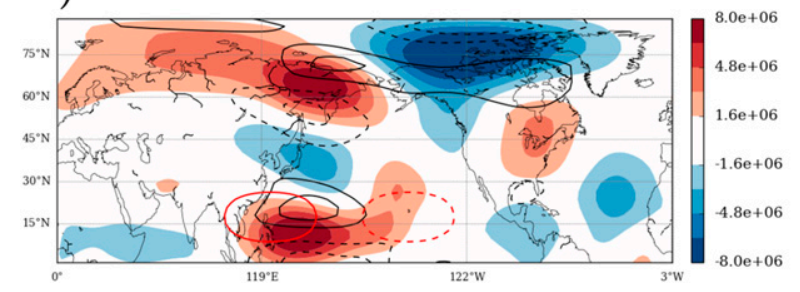

b)

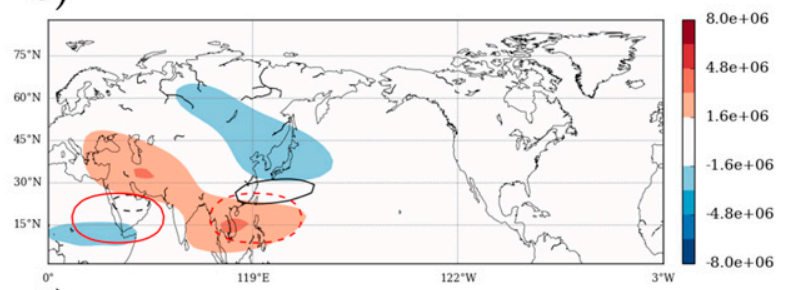

d)

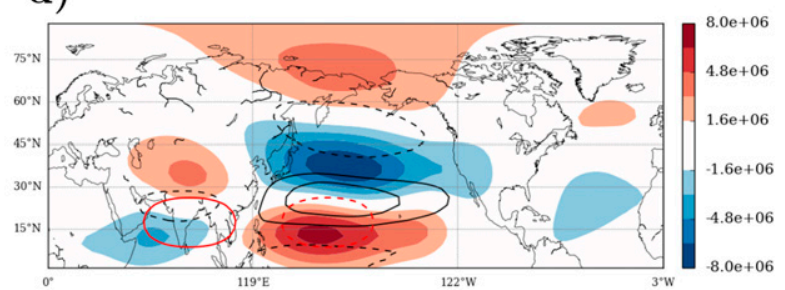

f)

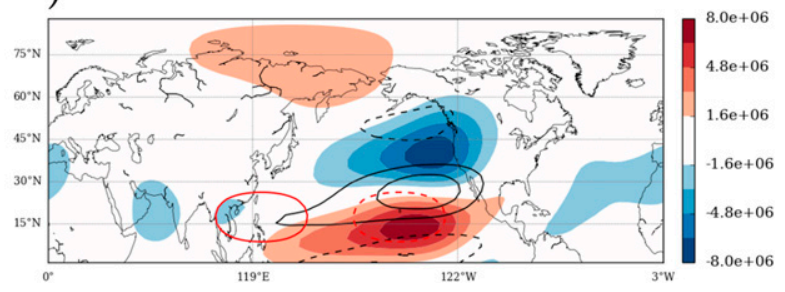

FIG. 14. The 200-mb streamfunction anomalies for the MJO phase 6 experiment with (left) a constant and (right) a time-varying reference flow at $t=$ (a),(b) 5, (c),(d) 15, and (e),(f) 25 days. The MJO forcing is moving eastward and has an amplitude $S_{0}=$ $-4 \times 10^{-11} \mathrm{~s}^{-2}$.

following MJO phase 6. Within the framework of our modeling approach, we can investigate that situation very simply by considering negative MJO forcing amplitudes. In this section, we focus on the case $S_{0}=-4 \times 10^{-11} \mathrm{~s}^{-2}$, which we call phase $6 \mathrm{MJO}$ forcing. Not surprisingly, we find streamfunction anomalies that have opposite sign compared to the MJO phase 3 forcing (Fig. 14). This is consistent with the emitted stationary Rossby wave being largely in the linear regime. A careful comparison with Fig. 2 shows that the anomalies do not exactly mirror those obtained for MJO phase 3 forcing. This is an indication that some nonlinearities are at play in that case as recently noted by Lin and Brunet (2018). Zonal wind anomalies are also of opposite signs and the Pacific jet is shifted equatorward as a consequence, in agreement with the results of Moore et al. (2010). As for the case of positive forcing amplitudes, when the reference flow is time varying, we also find that both ridges and troughs have an eastward elongated structure that is unlike the case of the stationary reference flow. At late times in the evolution ( $t>15$ days), a trough forms south of Alaska. A dipolar structure emerges in the Atlantic basin, with a trough located in the southern North Atlantic basin and a ridge located south of Greenland. Such a dipole is indicative of a negative NAO phase. In agreement, the distribution of the first EOF PC at $t=25$ days is biased toward negative values compared to the reference case without MJO forcing (Fig. 15a). Its mean value amounts to about $18 \%$ of the PDF standard deviation and leads to an increase of the number of $\mathrm{NAO}-$ days from $N_{\mathrm{NAO}-} \sim$ 1600 to 2100 , that is, a relative increase of $24 \%$. This is slightly smaller than the NAO+ bias we found for phase 3 MJO forcing. When compared with that case, the time evolution of $N_{\mathrm{NAO}-}$ shows an earlier increase that saturates at $t>15$ days to a more moderate value (Fig. 15b). This should be contrasted with the steady increase of $N_{\mathrm{NAO}}+$ we found between $t=15$ and 25 days for phase 3 forcing. Although qualitatively similar to the case of MJO phase 3 forcing (see Fig. 4), there are thus some differences between the two cases that are again suggestions of some nonlinearities being at play. Overall, we conclude that our simulations show a significant bias toward the negative phase of the NAO 15 to 20 days after MJO phase 6 . This is in agreement with the findings of Cassou (2008).

\section{b. Sensitivity to the MJO forcing amplitude}

The results described in the previous sections have shown that the time-varying nature of the atmospheric flow modifies the MJO-excited stationary Rossby wave 
a)

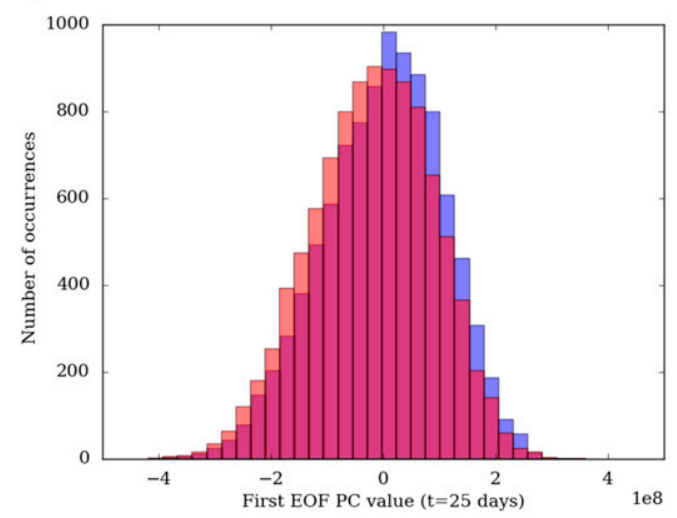

b)

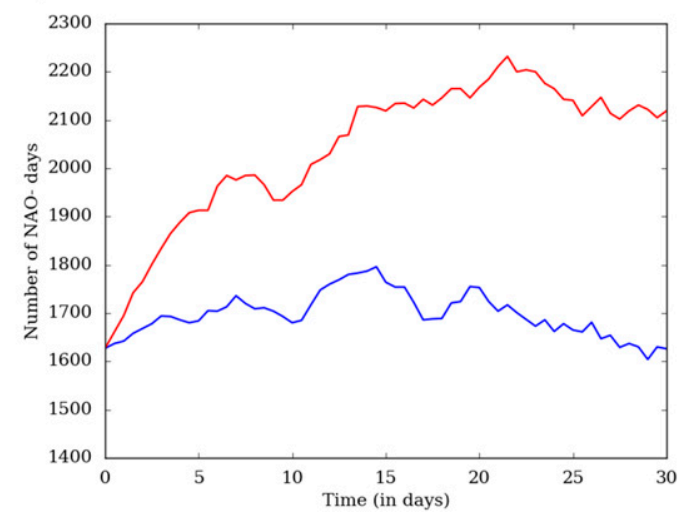

FIG. 15. As in Fig. 4, but for the case of phase 6-like MJO forcing and (b) the number of NAO- days.

path. For example, in the Pacific basin at time $t=15$ days, the anomalies are zonally elongated when the reference flow is time varying. We have shown that this is due to the modification of both the low- and high-frequency eddy-eddy interaction term in the streamfunction budget. This might suggest that the large amplitude of the MJO forcing is a necessary component of that modification because of the nonlinear nature of that term, and that we would recover the same stationary Rossby wave as in the absence of eddies (i.e., when the reference flow is stationary) for small enough MJO forcing amplitude. Here, we show this is not the case by considering a forcing amplitude $S_{0}=4 \times 10^{-13} \mathrm{~s}^{-2}$, that is, 100 times smaller than our basic configuration. We stress that the anomalies in this case are really tiny: for example, the zonal wind anomalies are on the order of a few centimeters per second. It is only because of the very large size of our ensemble of short runs that a significant averaged signal can be extracted. At $t=$ 15 days, we find that the streamfunction anomalies at $200 \mathrm{mb}$ are still different in the time-varying versus stationary reference flow experiments (Fig. 16). In addition, the spatial patterns of the anomalies in both cases are almost identical to those obtained with the finite-amplitude forcing (cf. the shadings and the contours, whose interval is scaled by two orders of magnitudes exactly compared to the shadings intervals): this is because the MJO-induced stationary Rossby wave is essentially in the linear regime and its amplitude scales linearly with the forcing amplitude with only minor modifications of its shape. We conclude that eddies modify the Rossby wave path regardless of the amplitude of the MJO forcing. Of course, this is because the nonlinear term $\delta \xi_{3}$ in the streamfunction budget is modified linearly by the stationary Rossby wave. If we denote by $\delta q_{i}^{H}$ and $\delta \psi_{i}^{H}$ the small modifications of the high-frequency $\mathrm{PV}$ and streamfunction at level $i$ due to the MJO, then $\delta \xi_{3}$ can formally be written as

$$
\delta \xi_{3}=\nabla^{-2} \sum_{i=1}^{3}\left[J\left(\delta q_{i}^{H}, \psi_{i}^{H}\right)+J\left(q_{i}^{H}, \delta \psi_{i}^{H}\right)\right]
$$

which renders explicit the linear nature of that term with respect to the forcing and explains our results.

However, this discussion should not hide the fact that some nonlinearities are at play (as discussed already when comparing our results for phase 3 and phase $6 \mathrm{MJO}$ forcings), and may potentially become more and more important as the MJO amplitude increases. To illustrate that point, we performed two additional series of shorts runs with $S_{0}=2 \times 10^{-11} \mathrm{~s}^{-2}$ and $S_{0}=6 \times 10^{-11} \mathrm{~s}^{-2}$. For each values of $S_{0}$, We next defined the number of excess $\mathrm{NAO}+$ days as

$$
\Delta N_{\mathrm{NAO}+}^{\text {excess }}=N_{\mathrm{NAO}+}\left(S_{0}\right)-N_{\mathrm{NAO}+}\left(S_{0}=0\right) .
$$

As expected, $\Delta N_{\mathrm{NAO}+}^{\text {excess }}$ increases with $S_{0}$ (filled blue circles in Fig. 17). Its variations with the MJO forcing amplitude can be fitted with the power law $\Delta N_{\mathrm{NAO}+}^{\text {excess }} \propto S_{0}^{\alpha}$ with $\alpha=1.24$ (dashed line in Fig. 17). The fact that $\alpha$ is larger than one highlights the effect of the flow nonlinearities on the NAO+ bias. One can further ask whether such a nonlinear behavior is coming from a nonlinear sensitivity of the mean PC to the forcing amplitude or from a change in the shape of the PC distribution. To address that point, we computed the number of excess $\mathrm{NAO}+$ days by assuming a Gaussian shape for the PC distribution with a standard deviation identical to the case without $\mathrm{MJO}$ forcing and considering a shift of the mean PC of $\delta_{\mathrm{PC}}$. This is given by

$$
\Delta N_{\mathrm{NAO}+}^{\text {expected }}=\frac{N}{2}\left[\operatorname{erf}\left(\frac{1}{\sqrt{2}}\right)-\operatorname{erf}\left(\frac{1-\delta_{\mathrm{PC}}}{\sqrt{2}}\right)\right]
$$


a)

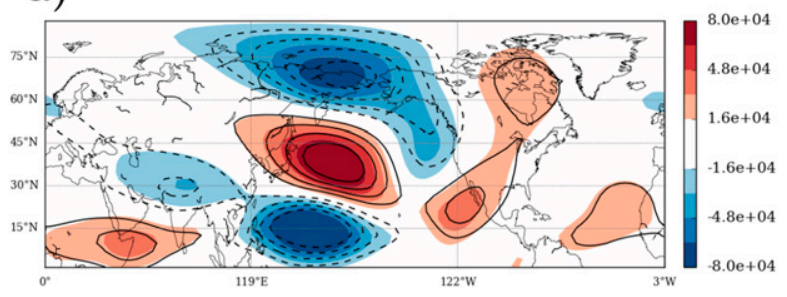

b)

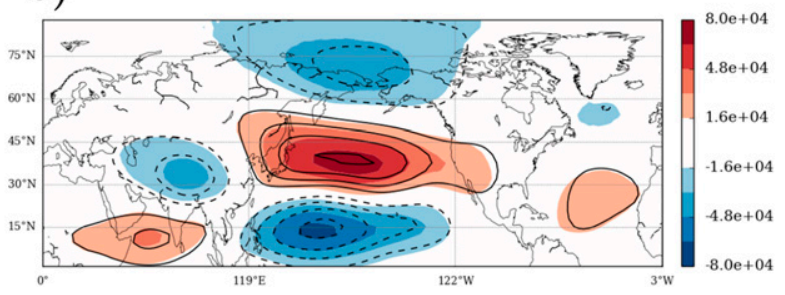

FIG. 16. (a) The 200-mb streamfunction anomalies at 15 days for an eastward-moving MJO forcing with amplitude $S_{0}=2 \times 10^{-13} \mathrm{~s}^{-2}$ (shading) and $S_{0}=4 \times 10^{-11} \mathrm{~s}^{-2}$ (black contours; CI: $1.875 \times 10^{5} \mathrm{~m}^{2} \mathrm{~s}^{-1}$; negative contours are dashed; zero contour is omitted) for the case of a stationary reference flow. (b) As in (a), but for the case of a time-varying reference flow.

where $\operatorname{erf}(\cdot)$ stands for the error function and $N=9967$ is the number of members of our series of runs. We made two estimates of $\Delta N_{\mathrm{NAO}+}^{\text {expecte }}$. One with the real shift in $\delta_{\mathrm{PC}}$ (red dotted line), the other with a shift in $\delta_{\mathrm{PC}}$, which linearly increases with the forcing amplitude. Its slope is evaluated from the results we obtained for the smallest MJO amplitude we considered (blue dotted line). The red dotted line is below the dashed blue line and has a power law with $\alpha=$ 1.25. The blue dotted line is below the red dotted line and the exponent of the fit is $\alpha=1.07$. This indicates that the nonlinearities are not only due to a faster than linear increase of the mean PC with the MJO forcing, but also to a modification of the shape of the PC distribution, both of which contribute similarly to the nonlinear increase of the number of $\mathrm{NAO}+$ excess days with the forcing amplitude.

\section{Conclusions}

The paper investigated the influence of the MJO on the NAO by performing numerical experiments with a dry three-level quasigeostrophic model on the sphere. To our knowledge, it is the most simplified model shown to reproduce the main properties of the relationship between the MJO and NAO seen in the observations. Since the model is relevant for midlatitude atmospheric dynamics only, the MJO is here reproduced by prescribing potential vorticity anomalies in the tropical and subtropical regions. This is different from the studies of Lin and Brunet (2018) and Shao et al. (2019), who both prescribed MJO-like diabatic heating anomalies in their dry primitive equation atmospheric models. One advantage of the quasigeostrophic model is its low computational cost that allowed us to perform a very large ensemble of simulations (9967 overall) and led to statistically robust results. Another advantage is that the PV formulation of the model made possible the development and analysis of a detailed PV budget. Finally, other main originalities of the present study concern the effect of stationary versus time-varying reference flows, fixed versus eastward-moving MJO-like forcing anomalies and linear as opposed to nonlinear processes.
In the most realistic setup we used, that is for a timevarying reference flow and an eastward-moving finiteamplitude MJO forcing, the following sequence of events was found during MJO-like phase 3. First, a quasistationary Rossby wave is excited in the tropics leading to a poleward displacement of the western Pacific jet. This modifies the transient wave activity, especially the synoptic one, in such a way that a ridge is formed south of Alaska. From the ridge sector toward the Atlantic sector, synoptic waves propagate more equatorward than in the case without MJO, which favors poleward eddy momentum fluxes in the Atlantic and increases the frequency of occurrence of the positive phase of the NAO. A PV budget confirms that it is the nonlinear interactions among the transient eddies, and thus the underlying wave-breaking processes, that are mainly responsible for setting the positive NAO phase. The situation is essentially reversed following phase 6 of the MJO and conducive to the negative phase of the NAO. For realistic amplitudes of the MJO-like forcing, we found increases in both NAO phases to be around $30 \%$ after 25 days, in reasonable agreement with the observations given the model simplicity. Additional information about the underlying processes can be summarized as follows:

- In the case of a stationary reference flow, the quasistationary Rossby wave in the eastern Pacific is less zonally oriented than in the case of the time-varying reference flow. There is no ridge south of Alaska during phase 3 and there is almost no projection of the anomalies over the Atlantic Ocean onto the NAO.

- The case of fixed MJO-like phase 3 is not so different from the eastward-moving one. The anomalies in the western Pacific are stronger and the ridge in the eastern Pacific is a bit weaker. The projection onto the NAO after 25 days is similar between the two cases. As noted by Shao et al. (2019), this is somewhat longer than the 15-day lag suggested by the observations. Such a difference can be attributed to the artificial initial flow in this experiment that lacks the influence of the previous MJO phases. By contrast, for the 


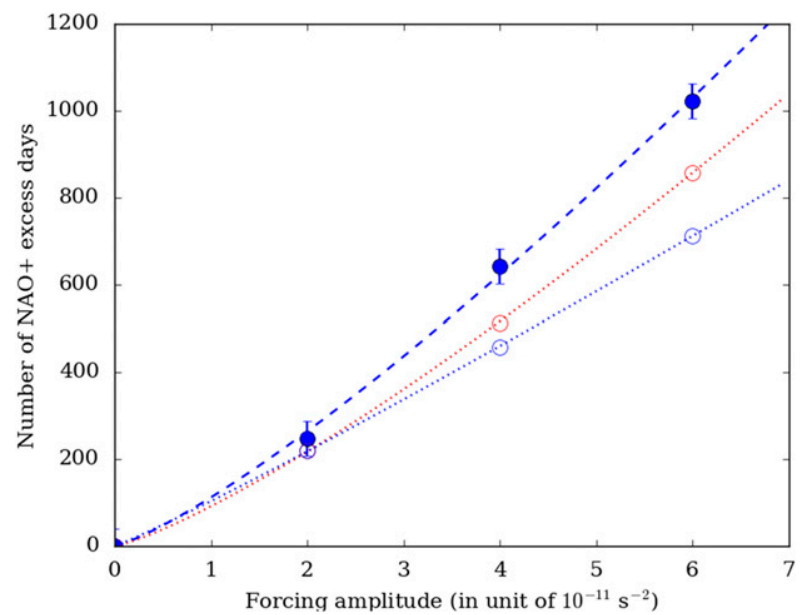

FIG. 17. Number of $\mathrm{NAO}+$ excess days $\Delta N_{\mathrm{NAO}+}^{\text {excess }}$ averaged between 25 and 30 days as a function of $S_{0}$ for the time-varying reference flow with an eastward-moving MJO forcing (filled blue symbol). The dashed blue line is a power-law fit to the simulations results. The red open circles show an estimate of $\Delta N_{\mathrm{NAO}+}^{\text {excess }}$ based on the diagnosed shift of the mean PC, assuming a Gaussian distribution of the PCs. The blue open circle is a linear estimate of $\Delta N_{\mathrm{NAO}+}^{\text {exces }}$ based on a linear extrapolation of the mean PC from its value when $S_{0}=2 \times 10^{-11} \mathrm{~s}^{-2}$. The red and blue dotted lines are power-law fits to the red and blue open circles, respectively.

eastward-moving $\mathrm{MJO}$ forcing case, the positive bias of the NAO occurs $\sim 10$ days after the MJO forcing crosses the position $\lambda \sim 110^{\circ}$ that corresponds to the fixed forcing experiments, in better agreement with the observed lagged correlations between the MJO and the NAO.

- At early times ( $t=5$ days), linear terms explain most of the projection onto the NAO and can be associated with a westward-moving Rossby wave. This is in agreement with the results of Lin and Brunet (2018). At latter times, the importance of these linear terms decreases and we find that they account for about $1 / 3$ to $1 / 2$ only of the projection onto the NAO (see, e.g., Fig. 6).

- Some nonlinearities are at play in the relationship between the MJO and the NAO. First, in agreement with Lin and Brunet (2018), the anomalies created by phases 3 and 6 are not entirely opposite. This is a signature of the influence of the flow synoptic activity: although the MJO forcing is symmetric between the two phases, the modifications to the background flow are not. The Pacific jet is deflected northward following phase 3 forcing but is more zonal following phase 6 forcing. As a result, the modifications of the synoptic eddies are not symmetric (Drouard et al. 2013), which explains the asymmetries obtained between the two phases. Second, by increasing the amplitude of the forcing of phase 3 , the increase in $\mathrm{NAO}+$ occurrence is more rapid than would be predicted based on a linear scaling.

The mechanism linking the North Pacific and North Atlantic sectors underlined in the present study supports other interbasin teleconnection studies such as those of Drouard et al. (2015) and Schemm et al. (2018) concerning the influence of ENSO in the North Atlantic sector and Tan et al. (2017) about the interpretation of the Western Hemisphere circulation pattern, all of which highlight the importance of a ridge in the eastern Pacific for setting up these teleconnections between the two basins.

Some limits of our analysis should be mentioned. First, the QG model is not appropriate to look at equatorial dynamics and the simulated quasi-stationary wave triggered by the MJO-like forcing may not be fully realistic. For example, we have shown that the forcing we use is associated with an instantaneous streamfunction forcing over the Atlantic and we have highlighted a spurious effect it might have on the linear Rossby wave that propagates toward that region. Future studies should look at the effect of similar MJO-like forcings in dry GCM to compare with the present quasigeostrophic framework. Using such an approach, Zheng and Chang (2019) recently obtained results that disagree with ours in two ways: first, they did not recover the MJO-NAO lagged relationship in their simulations. Second, a stationary wave model gives similar results to their fully evolving reference flow model. Understanding these differences with our work clearly requires further analysis. The comparison between our results and reanalysis data, although qualitative, also highlighted some potential deficiencies of our modeling strategy: at early times ( $t=15$ days), the Rossby wave over the Pacific always presents a more zonally elongated structure than observed. The reasons for this discrepancy should be further investigated, but a promising possibility is that it could be linked to the Pacific storm-track entrance. The latter is indeed poorly simulated over the Himalayas as a result of the very crude treatment of the topography in our QG model. Finally, our model only includes tropospheric dynamics and this excludes the possibility of analyzing the stratospheric pathway such as underlined by Garfinkel et al. (2014). Here again, more realistic numerical experiments made with GCMs are required to investigate the relative importance of the tropospheric and stratospheric pathways.

Acknowledgments. The authors acknowledge funding from the ANR via its Belmont Forum call (Project GOTHAM; ANR-15-JCLI-0004). 


\section{APPENDIX}

\section{Forcing Function Calculation}

Here we describe how we calculate the forcing function used throughout this paper in the time-varying runs. The problem can be formulated as finding the function $S_{i}^{\text {clim }}(\lambda, \phi)$, for each pressure levels $i$, for which the model climatology is closest to the observations. For that purpose, we define the function $\mathscr{F}$ according to

$$
\mathscr{F}: S_{i} \mapsto \bar{q}-\bar{q}^{\mathrm{obs}},
$$

where $\bar{q}$ corresponds to the climatological PV that results from running the QG model with the forcing function $S_{i}$ and $\bar{q}^{\text {obs }}$ is the observed PV climatology. Finding $S_{i}^{\text {clim }}$ thus amounts to finding the root of the function $\mathscr{F}$. The difficulties arise from the fact that $\mathscr{F}$ is only implicitly defined and is a function of many variables, namely, the model resolution. This makes standard zero finding algorithms, such as the Newton-Raphtson method, not directly applicable. Instead, we implemented a Newton-Krylov algorithm (Knoll and Keyes 2004) because it is known to be more efficient for such large problems. In addition, the loose generalized minimum residual method (LGMRES; Baker et al. 2005) is used to estimate the Jacobian associated with the $\mathscr{F}$ function.

In practice, we averaged daily ERA-Interim data over the period 1979-2017 to compute $\bar{q}^{\text {obs }}$ and we used the newton_krylov function available through the SciPy python package (https://docs.scipy.org/doc/scipy-0.14.0/ reference/generated/scipy.optimize.newton_krylov.html) to compute $S_{i}^{\text {clim }}$. Each iteration of the algorithm requires to evaluate the model climatology several times for different forcing functions. To reduce the computational burden, this is done by running the model for 1000 days only, averaging the PV over the last 700 days. The closeness of the model climatology to the observations is then evaluated using the $\mathscr{L}^{2}$ norm for the PV:

$$
\mathscr{L}_{2}(\bar{q})=\sqrt{\sum_{\text {cells }}\left(\bar{q}-\bar{q}^{\mathrm{obs}}\right)^{2}}
$$

where the sum is taken over the entire number of cells of the domain. ${ }^{\mathrm{A} 1}$ We find that $\mathscr{L}_{2}(\bar{q})$ quickly decreases after a few iterations and saturates to a low but finite value after about 10 iterations (Fig. A1). The existence of such a lower limit to the norm we calculate is partly due to the finite duration of the model integration that

\footnotetext{
${ }^{\text {A1 }}$ The norm we used does not weight each cell according to its area. This is to account for the fact that the polar regions were found to be the most difficult regions to converge.
}

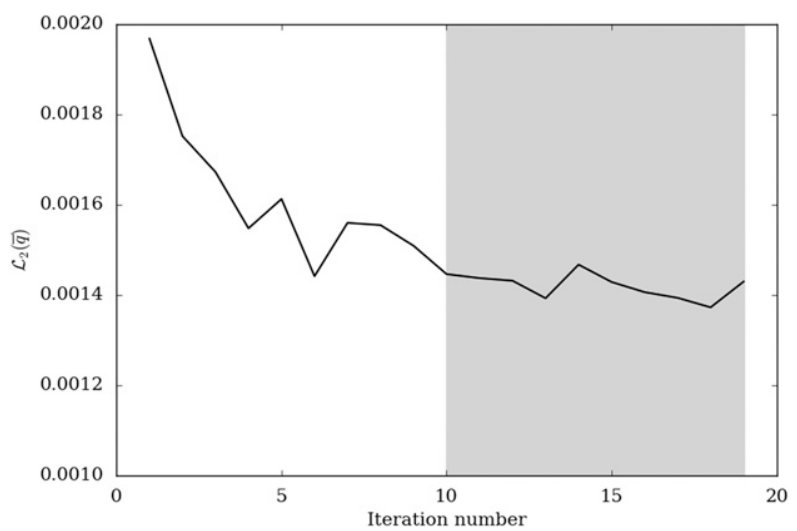

FIG. A1. Evolution of $\mathscr{L}_{2}(\bar{q})$ with the iteration number when using the Newton-Krylov algorithm to estimate $S_{i}^{\text {clim. }}$. The gray shaded area corresponds to those iterations over which the forcing function is averaged.

leads to errors in evaluating the model climatology, but also to the low realism of the model itself that limits its ability to accurately reproduce the observations. For these reasons, we decided to calculate $S_{i}^{\text {clim }}$ as the average of the forcing functions obtained between the iterations 10 and 19 (gray shading in Fig. A1). Although arbitrary, such a choice makes the iterative procedure robust and reproducible. For a given observed climatology, the total procedure requires about 5 days of $\mathrm{CPU}$ time on typical workstations.

\section{REFERENCES}

Ambrizzi, T., and B. J. Hoskins, 1997: Stationary Rossby-wave propagation in a baroclinic atmosphere. Quart. J. Roy. Meteor. Soc., 123, 919-928, https://doi.org/10.1002/qj.49712354007.

Baker, A. H., E. R. Jessup, and T. Manteuffel, 2005: A technique for accelerating the convergence of restarted GMRES. SIAM J. Matrix Anal. Appl., 26, 962-984, https://doi.org/10.1137/ S0895479803422014.

Bjerknes, J., 1966: A possible response of the atmospheric Hadley circulation to equatorial anomalies of ocean temperature. Tellus, 18A, 820-829, https://doi.org/10.1111/j.2153-3490.1966.tb00303.x.

Cassou, C., 2008: Intraseasonal interaction between the MaddenJulian oscillation and the North Atlantic Oscillation. Nature, 455, 523-527, https://doi.org/10.1038/nature07286.

Cayan, D. R., K. T. Redmond, and L. G. Riddle, 1999: ENSO and hydrologic extremes in the western United States. J. Climate, 12, 2881-2893, https://doi.org/10.1175/1520-0442(1999)012<2881: EAHEIT > 2.0.CO;2.

Chang, E. K. M., 2006: An idealized nonlinear model of the Northern Hemisphere winter storm tracks. J. Atmos. Sci., 63, 1818-1839, https://doi.org/10.1175/JAS3726.1.

Corti, S., A. Giannini, S. Tibaldi, and F. Molteni, 1997: Patterns of lowfrequency variability in a three-level quasi-geostrophic model. Climate Dyn., 13, 883-904, https://doi.org/10.1007/s003820050203.

Curtis, S., and D. W. Gamble, 2016: The boreal winter MaddenJulian oscillation's influence on summertime precipitation in the greater Caribbean. J. Geophys. Res. Atmos., 121, 7592-7605, https://doi.org/10.1002/2016JD025031. 
Domeisen, D. I. V., C. I. Garfinkel, and A. H. Butler, 2019: The teleconnection of El Niño Southern Oscillation to the stratosphere. Rev. Geophys., 57, 5-47, https://doi.org/10.1029/ 2018RG000596.

Drouard, M., G. Rivière, and P. Arbogast, 2013: The North Atlantic Oscillation response to large-scale atmospheric anomalies in the northeastern Pacific. J. Atmos. Sci., 70, 2854-2874, https://doi.org/10.1175/JAS-D-12-0351.1.

,$- \ldots$, and $\longrightarrow, 2015$ : The link between the North Pacific climate variability and the North Atlantic Oscillation via downstream propagation of synoptic waves. J. Climate, $\mathbf{2 8}$, 3957-3976, https://doi.org/10.1175/JCLI-D-14-00552.1.

Frederiksen, J. S., and H. Lin, 2013: Tropical-extratropical interactions of intraseasonal oscillations. J. Atmos. Sci., 70, 3180-3197, https://doi.org/10.1175/JAS-D-12-0302.1.

Garfinkel, C. I., S. B. Feldstein, D. W. Waugh, C. Yoo, and S. Lee, 2012: Observed connection between stratospheric sudden warmings and the Madden-Julian oscillation. Geophys. Res. Lett., 39, L18807, https://doi.org/10.1029/2012GL053144.

— J. J. Benedict, and E. D. Maloney, 2014: Impact of the MJO on the boreal winter extratropical circulation. Geophys. Res. Lett., 41, 6055-6062, https://doi.org/10.1002/2014GL061094.

Gershunov, A., and T. P. Barnett, 1998: ENSO influence on intraseasonal extreme rainfall and temperature frequencies in the contiguous United States: Observations and model results. J. Climate, 11, 1575-1586, https://doi.org/10.1175/ 1520-0442(1998)011<1575:EIOIER > 2.0.CO;2.

Gill, A. E., 1980: Some simple solutions for heat-induced tropical circulation. Quart. J. Roy. Meteor. Soc., 106, 447-462, https:// doi.org/10.1002/qj.49710644905.

Goss, M., and S. B. Feldstein, 2015: The impact of the initial flow on the extratropical response to Madden-Julian oscillation convective heating. Mon. Wea. Rev., 143, 1104-1121, https:// doi.org/10.1175/MWR-D-14-00141.1.

_, and —_, 2017: Why do similar patterns of tropical convection yield extratropical circulation anomalies of opposite sign? J. Atmos. Sci., 74, 487-511, https://doi.org/10.1175/ JAS-D-16-0067.1.

$\longrightarrow$, and — 2018: Testing the sensitivity of the extratropical response to the location, amplitude, and propagation speed of tropical convection. J. Atmos. Sci., 75, 639-655, https://doi.org/ 10.1175/JAS-D-17-0132.1.

Guo, Y., T. Shinoda, J. Lin, and E. K. M. Chang, 2017: Variations of Northern Hemisphere storm track and extratropical cyclone activity associated with the MaddenJulian oscillation. J. Climate, 30, 4799-4818, https://doi.org/ 10.1175/JCLI-D-16-0513.1.

Hall, N. M. J., 2000: A simple GCM based on dry dynamics and constant forcing. J. Atmos. Sci., 57, 1557-1572, https://doi.org/ 10.1175/1520-0469(2000)057<1557:ASGBOD>2.0.CO;2.

Held, I. M., S. W. Lyons, and S. Nigam, 1989: Transients and the extratropical response to El Niño. J. Atmos. Sci., 46, 163-174, https:// doi.org/10.1175/1520-0469(1989)046<0163:TATERT>2.0.CO;2.

Henderson, G. R., B. S. Barrett, and D. M. Lafleur, 2014: Arctic sea ice and the Madden-Julian oscillation (MJO). Climate Dyn., 43, 2185-2196, https://doi.org/10.1007/s00382-013-2043-y.

Henderson, S. A., E. D. Maloney, and E. A. Barnes, 2016: The influence of the Madden-Julian oscillation on Northern Hemisphere winter blocking. J. Climate, 29, 4597-4616, https://doi.org/10.1175/JCLI-D-15-0502.1.

Hoerling, M. P., and A. Kumar, 2002: Atmospheric response patterns associated with tropical forcing. J. Climate, 15, 2184-2203, https:// doi.org/10.1175/1520-0442(2002)015<2184:ARPAWT>2.0.CO;2.
Hoskins, B. J., and D. J. Karoly, 1981: The steady linear response of a spherical atmosphere to thermal and orographic forcing. J. Atmos. Sci., 38, 1179-1196, https://doi.org/10.1175/ 1520-0469(1981)038<1179:TSLROA > 2.0.CO;2.

_ , and F.-F. Jin, 1991: The initial value problem for tropical perturbations to a baroclinic atmosphere. Quart. J. Roy. Meteor. Soc., 117, 299-317, https://doi.org/10.1002/qj.49711749803.

__ , and T. Ambrizzi, 1993: Rossby wave propagation on a realistic longitudinally varying flow. J. Atmos. Sci., 50, 1661-1671, https://doi.org/10.1175/1520-0469(1993)050<1661: RWPOAR $>2.0 . \mathrm{CO} ; 2$.

Jiang, Z., S. B. Feldstein, and S. Lee, 2017: The relationship between the Madden-Julian oscillation and the North Atlantic Oscillation. Quart. J. Roy. Meteor. Soc., 143, 240-250, https:// doi.org/10.1002/qj.2917.

Jin, F., and B. J. Hoskins, 1995: The direct response to tropical heating in a baroclinic atmosphere. J. Atmos. Sci., 52, 307-319, https:// doi.org/10.1175/1520-0469(1995)052<0307:TDRTTH>2.0.CO;2.

Kang, W., and E. Tziperman, 2018: The MJO-SSW teleconnection: Interaction between MJO-forced waves and the midlatitude jet. Geophys. Res. Lett., 45, 4400-4409, https://doi.org/10.1029/ 2018 GL077937.

Kiladis, G. N., K. H. Straub, and P. T. Haertel, 2005: Zonal and vertical structure of the Madden-Julian oscillation. J. Atmos. Sci., 62, 2790-2809, https://doi.org/10.1175/JAS3520.1.

Knoll, D. A., and D. E. Keyes, 2004: Jacobian-free Newton-Krylov methods: A survey of approaches and applications. J. Comput. Phys., 193, 357-397, https://doi.org/10.1016/j.jcp.2003.08.010.

Lanczos, C., 1988: Applied Analysis. Courier Corporation, 539 pp.

Lee, S., T. Gong, N. Johnson, S. B. Feldstein, and D. Pollard, 2011: On the possible link between tropical convection and the Northern Hemisphere Arctic surface air temperature change between 1958 and 2001. J. Climate, 24, 4350-4367, https:// doi.org/10.1175/2011JCLI4003.1.

L'Heureux, M. L., and D. W. J. Thompson, 2006: Observed relationships between the El Niño-Southern Oscillation and the extratropical zonal-mean circulation. J. Climate, 19, 276-287, https://doi.org/10.1175/JCLI3617.1.

_ , and R. W. Higgins, 2008: Boreal winter links between the Madden-Julian oscillation and the Arctic Oscillation. J. Climate, 21, 3040-3050, https://doi.org/10.1175/2007JCLI1955.1.

Lin, H., and Z. Wu, 2012: Indian summer monsoon influence on the climate in the North Atlantic-European region. Climate Dyn., 39, 303-311, https://doi.org/10.1007/s00382-011-1286-8.

— Nonlinearity and sensitivity to the initial state. J. Atmos. Sci., 75, 219-234, https://doi.org/10.1175/JAS-D-17-0189.1.

$\longrightarrow,-$, and J. Derome, 2009: An observed connection between the North Atlantic Oscillation and the Madden-Julian oscillation. J. Climate, 22, 364-380, https://doi.org/10.1175/2008JCLI2515.1.

,-- , and R. Mo, 2010: Impact of the Madden-Julian oscillation on wintertime precipitation in Canada. Mon. Wea. Rev., 138, 3822-3839, https://doi.org/10.1175/2010MWR3363.1.

Lukens, K. E., S. B. Feldstein, C. Yoo, and S. Lee, 2017: The dynamics of the extratropical response to Madden-Julian oscillation convection. Quart. J. Roy. Meteor. Soc., 143, 1095-1106, https://doi.org/10.1002/qj.2993.

Lunkeit, F., K. Fraedrich, and S. E. Bauer, 1998: Storm tracks in a warmer climate: Sensitivity studies with a simplified global circulation model. Climate Dyn., 14, 813-826, https://doi.org/ 10.1007/s003820050257.

Madden, R. A., and P. R. Julian, 1971: Detection of a 40-50 day oscillation in the zonal wind in the tropical Pacific. J. Atmos. 
Sci., 28, 702-708, https://doi.org/10.1175/1520-0469(1971) 028<0702:DOADOI $>2.0 . \mathrm{CO} ; 2$.

Mak, M., 1991: Influences of the Earth's sphericity in the quasigeostrophic theory. J. Meteor. Soc. Japan, 69, 497-511, https:// doi.org/10.2151/jmsj1965.69.5_497.

Marshall, J., and F. Molteni, 1993: Toward a dynamical understanding of planetary-scale flow regimes. J. Atmos. Sci., 50, 1792-1818, https://doi.org/10.1175/1520-0469(1993)050<1792: TADUOP $>2.0 . \mathrm{CO} ; 2$.

Matthews, A. J., and G. N. Kiladis, 1999: The tropical extratropical interaction between high-frequency transients and the MaddenJulian oscillation. Mon. Wea. Rev., 127, 661-677, https://doi.org/ 10.1175/1520-0493(1999)127<0661:TTEIBH > 2.0.CO;2.

_ J. B. Hoskins, and M. Masutani, 2004: The global response to tropical heating in the Madden-Julian oscillation during the northern winter. Quart. J. Roy. Meteor. Soc., 130, 1991-2011, https://doi.org/10.1256/qj.02.123.

Moore, R. W., O. Martius, and T. Spengler, 2010: The modulation of the subtropical and extratropical atmosphere in the Pacific basin in response to the Madden-Julian oscillation. Mon. Wea. Rev., 138, 2761-2779, https://doi.org/10.1175/2010MWR3194.1.

Perdigón-Morales, J., R. Romero-Centeno, B. S. Barrett, and P. Ordoñez, 2019: Intraseasonal variability of summer precipitation in Mexico: MJO influence on the midsummer drought. J. Climate, 32, 2313-2327, https://doi.org/10.1175/ JCLI-D-18-0425.1.

Sakaeda, N., and P. E. Roundy, 2014: The role of interactions between multiscale circulations on the observed zonally averaged zonal wind variability associated with the Madden-Julian oscillation. J. Atmos. Sci., 71, 3816-3836, https://doi.org/ 10.1175/JAS-D-13-0304.1.

Sardeshmukh, P. D., and B. J. Hoskins, 1988: The generation of global rotational flow by steady idealized tropical divergence. J. Atmos. Sci., 45, 1228-1251, https://doi.org/10.1175/15200469(1988)045<1228:TGOGRF $>2.0$.CO;2.

Schemm, S., G. Rivière, L. M. Ciasto, and C. Li, 2018: Extratropical cyclogenesis changes in connection with tropospheric ENSO teleconnections to the North Atlantic: Role of stationary and transient waves. J. Atmos. Sci., 75, 3943-3964, https://doi.org/ 10.1175/JAS-D-17-0340.1.

Seo, K.-H., and S.-W. Son, 2012: The global atmospheric circulation response to tropical diabatic heating associated with the Madden-Julian oscillation during northern winter. J. Atmos. Sci., 69, 79-96, https://doi.org/10.1175/2011JAS3686.1.

, H.-J. Lee, and D. M. W. Frierson, 2016: Unraveling the teleconnection mechanisms that induce wintertime temperature anomalies over the Northern Hemisphere continents in response to the MJO.J. Atmos. Sci., 73, 3557-3571, https://doi.org/ 10.1175/JAS-D-16-0036.1.

Shao, X., J. Song, and S. Li, 2019: The lagged connection of the positive NAO with the MJO phase 3 in a simplified atmospheric model. Theor. Appl. Climatol., 135, 1091-1103, https:// doi.org/10.1007/s00704-018-2425-5.

Stan, C., D. M. Straus, J. S. Frederiksen, H. Lin, E. D. Maloney, and C. Schumacher, 2017: Review of tropical-extratropical teleconnections on intraseasonal time scales. Rev. Geophys., 55 , 902-937, https://doi.org/10.1002/2016RG000538.

Straus, D. M., E. Swenson, and C.-L. Lappen, 2015: The MJO cycle forcing of the North Atlantic circulation: Intervention experiments with the Community Earth System Model. J. Atmos. Sci., 72, 660-681, https://doi.org/10.1175/JAS-D-14-0145.1.

Tan, X., M. Bao, D. L. Hartmann, and P. Ceppi, 2017: The role of synoptic waves in the formation and maintenance of the Western Hemisphere circulation pattern. J. Climate, 30, 10 259-10 274, https://doi.org/10.1175/JCLI-D-17-0158.1.

Trenberth, K. E., 1986: An assessment of the impact of transient eddies on the zonal flow during a blocking episode using localized Eliassen-Palm flux diagnostics. J. Atmos. Sci., 43, 2070-2087, https://doi.org/10.1175/1520-0469(1986)043<2070: AAOTIO $>2.0 . \mathrm{CO} ; 2$

Wheeler, M. C., and H. H. Hendon, 2004: An all-season real-time multivariate MJO index: Development of an index for monitoring and prediction. Mon. Wea. Rev., 132, 1917-1932, https://doi.org/ 10.1175/1520-0493(2004)132<1917:AARMMI>2.0.CO;2.

Yuan, X., M. R. Kaplan, and M. A. Cane, 2018: The interconnected global climate system-A review of tropical-polar teleconnections. J. Climate, 31, 5765-5792, https://doi.org/10.1175/ JCLI-D-16-0637.1.

Yuval, J., and Y. Kaspi, 2016: Eddy activity sensitivity to changes in the vertical structure of baroclinicity. J. Atmos. Sci., 73, 1709-1726, https://doi.org/10.1175/JAS-D-15-0128.1.

Zhang, C., 2005: Madden-Julian oscillation. Rev. Geophys., 43, RG2003, https://doi.org/10.1029/2004RG000158.

Zheng, C., and E. K. M. Chang, 2019: The role of MJO propagation, lifetime, and intensity on modulating the temporal evolution of the MJO extratropical response. J. Geophys. Res. Atmos., 124, 5352-5378, https://doi.org/10.1029/2019JD030258.

- - — , H.-M. Kim, M. Zhang, and W. Wang, 2018: Impacts of the Madden-Julian oscillation on storm-track activity, surface air temperature, and precipitation over North America. J. Climate, 31, 6113-6134, https://doi.org/10.1175/ JCLI-D-17-0534.1. 